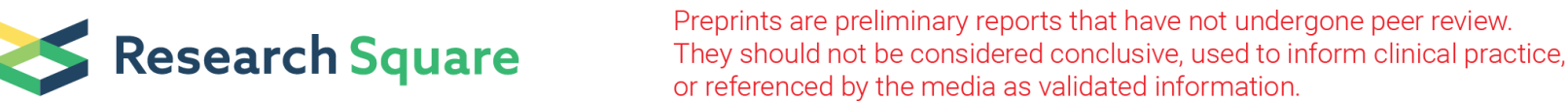

\section{Pyroelectric Catalysis-based "Nano-lymphatic" Reduces Tumor Interstitial Pressure for Enhanced Penetration and Hydrodynamic Therapy}

Yuchu He

Yanshan University

Zhuo Li

Yanshan University

Cong Cong

Yanshan University

Fei Ye

Yanshan University https://orcid.org/0000-0003-2480-4639

Jingyue Yang

Yanshan University

Xuwu Zhang

Yanshan University

Yi Yuan

Yanshan University

Zhenhe Ma

Northeastern University

Kaiqing Zhang

Yanshan University

Yang Lin

Northeastern University at Qinhuangdao

Lizhao Zheng

Northeastern University at Qinhuangdao

Dawei Gao ( $\nabla$ dwgao@ysu.edu.cn )

Yanshan University

Article

Keywords: Tumor interstitial pressure, Nano-lymphatic, Pyroelectric catalysis, Enhanced tumor penetration, Hydrodynamic therapy

Posted Date: February 4th, 2021 
DOI: https://doi.org/10.21203/rs.3.rs-156886/v1

License: (c) (1) This work is licensed under a Creative Commons Attribution 4.0 International License. Read Full License 


\section{Abstract}

Owing to deficiency of lymphatic reflux in the tumor, the retention of tumor interstitial fluid causes the aggravation of tumor interstitial pressure (TIP), which leads to unsatisfactory tumor penetration of nanomedicine. It is the main inducement of tumor recurrence and metastasis. Herein, we design a pyroelectric catalysis-based "Nano-lymphatic" to decrease the TIP for enhanced tumor penetration and treatments. It realizes photothermal therapy and decomposition of tumor interstitial fluid under NIR-II laser irradiation after reaching the tumor, which reduces the TIP for enhanced tumor penetration. Simultaneously, reactive oxygen species generated during the pyroelectric catalysis can further damage deep tumor stem cells. The results indicate that the "Nano-lymphatic" relieves $52 \%$ of TIP, leading to enhanced tumor penetration, which effectively inhibits the tumor proliferation (93.75\%) and recurrence. Our finding presents a novel strategy to reduce TIP by pyroelectric catalysis for enhanced tumor penetration and improved treatments, which is of great significance for drug delivery.

\section{Introduction}

Nowadays, drug delivery has become one of the most controversial issues, which is also the top concern of all anticancer therapies, with the rapid development of nanomedicines ${ }^{1-5}$. The major obstacle in achieving better delivery efficiency is the abnormally high tumor interstitial pressure (TIP) ${ }^{6-9}$. It forms a barrier to the efficient uptake of the therapeutic agents from blood capillaries. In human capillaries, the venous pressure is nearly $20 \mathrm{mmHg}$, while the interstitial pressure of normal tissues is negative (about -1 to $-3 \mathrm{mmHg})^{6}$. This outward pressure assures the penetration out from capillaries to the surrounding tissues. Whereafter, the lymphatic promotes the excess tissue fluid to flow back to the blood system, completing the blood circulation ${ }^{10-12}$. However, the interstitial pressure in the tumor is as high as 30 130 $\mathrm{mmHg}$ due to the retention of interstitial fluid caused by deficient lymph-vessel and lymphatic reflux, as well as dense extracellular matrix $(E C M)^{13-15}$. The much smaller net outward pressure or even net inward pressure seriously limits the normal flow from vessels into the tumor ${ }^{3}$, which leads to unsatisfactory delivery efficiency of therapeutic agents. Among the mounting works on drug delivery, most efforts focus on reducing tumor interstitial solid pressure (TISP) caused by dense ECM, proteins, and fibroblasts via enzymolysis, which left the mass deposition of interstitial fluid unsolved ${ }^{16-18}$. At present, little research can effectively reduce the tumor interstitial fluid pressure (TIFP). Therefore, it is still a big challenge to decrease the TIFP for efficient tumor penetration and treatments of nanomedicines.

Because the basis of tumor interstitial fluid is water (over $90 \%$ ), the decomposition of water can effectively reduce the volume of tumor interstitial fluid, meaning that decrease of TIFP ${ }^{19}$. Our group has designed a photocatalytic nanomedicine system to reduce TIFP, inspired by the catalytic water splitting employed in the field of new energy ${ }^{20}$. This nanomedicine mimics lymphatic in releasing tumor interstitial fluid and is termed as "Nano-lymphatic". In the previous report, we have preliminarily showcased the feasibility of the tumor interstitial fluid decomposition and decrease of TIFP by photocatalysis ${ }^{19}$. Nevertheless, the catalytic nanomedicine system could only be excited by the high-energy ultraviolet- 
visible light, which highly restricted its application in vivo due to the weak tissue penetration of ultravioletvisible light ${ }^{21-23}$. Therefore, we proposed an ideal and unexplored strategy to address the problem: Reducing TIP by pyroelectric catalysis. Different from photocatalysis, pyroelectric catalysis occurs under temperature variation instead of light excitation ${ }^{24,25}$. The pyro-generated positive and negative charges initiate the redox reactions, including water splitting and reactive oxygen species (ROS) generation ${ }^{26-28}$. Excitingly, the smart combination of photothermal agents and pyroelectric materials decomposes the tumor interstitial fluid to reduce TIFP via pyroelectric catalysis-based water splitting and ablates tumor ECM, proteins, and fibroblasts to reduce TISP via hyperpyrexia. Releasing of TIP in this way can improve the permeability of nanomedicine into the center of tumors significantly. Furthermore, the ROS generated during the pyroelectric catalysis can further damage the deep tumor stem cells ${ }^{29,30}$. We identify this tumor-penetrating therapeutic strategy based on water splitting and ROS generation as "Hydrodynamic therapy".

A well-designed pyroelectric catalysis-based "Nano-lymphatic" is reported in this study for enhanced tumor penetration by reducing TIP, which combines photothermal therapy (PTT) and "Hydrodynamic therapy". In detail, the pyroelectric material $\mathrm{CdS}$ is grown on an ultrathin $\mathrm{Nb}_{2} \mathrm{C}$ nanosheet (MXene) in situ to provide $\mathrm{M} / \mathrm{CdS}$, which is then modified with tumor-targeted hyaluronic acid (HA) to the final "Nanolymphatic" (M/CdS-HA). When the "Nano-lymphatic" reaches the tumor region through HA-mediated tumor target and under NIR-II (1064 nm) laser irradiation, MXene reduces the TISP via PTT first. The temperature variation then triggers $\mathrm{CdS}$ to decompose the tumor interstitial fluid via pyroelectric catalysis, leading to the decrease of TIFP. Benefit from the decrease of both TISP and TIFP, the "Nano-lymphatic" can highly penetrate the center of the tumor. Concurrently, the ROS generated during the pyroelectric catalysis breaks the ROS level threshold in the deep tumor stem cells, resulting in oxidative damage of cellular constituents to cell apoptosis or necrosis (Scheme 1). Our findings reveal an innovative way to enhance the tumor penetration of nanomedicine, which is hugely critical in drug delivery. It is the first demonstration of pyroelectric catalysis-based water splitting strategy for enhanced tumor penetration and improved treatments to the best of our knowledge.

Scheme 1. Illustration of the "Nano-lymphatic" (M/CdS-HA) synthesis and decrease of TIP by pyroelectric catalysis for enhanced tumor penetration, PTT, and "Hydrodynamic therapy". (see Supplemental Files for Scheme 1)

\section{Results}

Characterization. CdS was formed on the surface of MXene in situ for a close heterojunction (M/CdS). The morphologies of MXene and M/CdS were tested by transmission electron microscopy (TEM). As shown in Fig. 1a, after ultrasonic exfoliation, the multi-layer MXene was converted to ultrathin MXene. Besides, a large number of CdS nanoparticles were conspicuously dispersed on the ultrathin MXene to provide M/CdS. Modification with HA did not affect the structure and size of M/CdS. To further investigate the microtopography of M/CdS, high-resolution TEM (HRTEM) was employed. According to 
Fig. $1 \mathrm{~b}$, the lattice spacing of $0.262 \mathrm{~nm}$ and $0.357 \mathrm{~nm}$ corresponded to $\mathrm{Nb}_{2} \mathrm{C}$ and $\mathrm{CdS}$, respectively. Furthermore, the scanning TEM high-angle annular dark-field (STEM-HAADF) image and elements mapping of $\mathrm{M} / \mathrm{CdS}$ were revealed in Fig. 1c. The elements $\mathrm{C}$ and $\mathrm{Nb}$ corresponded to $\mathrm{MXene}$, and $\mathrm{Cd}$ and $S$ corresponded to CdS. The above results confirmed the successful synthesis of M/CdS. The atomic force microscope (AFM) was used to characterize the height of M/CdS. As revealed in Fig. 1d, the height of M/CdS was about $5.2 \mathrm{~nm}$, which further proved the ultrathin structure of M/CdS. This ultrathin structure was in favor of the transition of charge carriers. The size distribution and zeta potentials of MXene, CdS, and M/CdS were evaluated by dynamic light scattering (DLS). The mean size of CdS was about $10 \mathrm{~nm}$, while that of MXene and M/CdS were about $200 \mathrm{~nm}$ (Fig. 1e). This appropriate size of M/CdS is favorable for the tumor target. As revealed in Supplementary Fig. 1, the M/CdS showed a negative potential of about $-15 \mathrm{mV}$, which was further decreased to $-23 \mathrm{mV}$ in M/CdS-HA. Due to the negative charge, M/CdS-HA expressed reduced clearance and less absorption to the surface of cells than positively charged materials.

Pyroelectric effect. Schematic illustration of pyroelectric effect and relation between temperature variation and pyroelectric current was shown in Fig. 2a. Temperature variation can induce the motions of atoms in the pyroelectric material, leading to the change of polarization for a built-in pyroelectric field. When the temperature goes up $(\mathrm{dT} / \mathrm{dt}>0)$, the electric dipole moments decrease, which reduces the polarization intensity of pyroelectric material. The balance between the screening charges and polarization charges can be partially broken. Therefore, owing to the weak constraint, some screening charges is driven to the material surface to establish a new equilibrium. The pyro-generated free charges on the surface facilitate a pyroelectric potential through the electrode connected with an open circuit. If a short circuit alters the open circuit, the pyroelectric current will emerge ${ }^{25}$. On the contrary, when temperature decreases $(\mathrm{dT} / \mathrm{dt}<0)$, the heat dissipation from pyroelectric material leads to an increase of polarization intensity. Thus, the free charges from the surrounding electrolyte are adsorbed and redistributed to compensate for dipoles change. Likewise, an opposite pyroelectric current appears. When the temperature keeps invariant $(\mathrm{dT} / \mathrm{dt}=0)$, the electric balance in pyroelectric material is unbroken. Thus, none of pyroelectric potential and current generated ${ }^{31,32}$.

The electrochemical properties and pyroelectric effect of M/CdS were characterized by the electrochemical workstation. First, the electrochemical impedance spectra (EIS) Nyquist plots of CdS, MXene, and M/CdS in the dark and with 1064-nm laser irradiation were tested respectively, to evaluate the pyroelectric-induced electric charge transfer. As shown in Fig. 2b, the radii of semicircle for CdS showed no significant change in response to laser irradiation, which was reasoned that the CdS could not be excited by the low-energy 1064-nm laser ( $1.17 \mathrm{eV})$. Meanwhile, the radii of semicircle for MXene exhibited a slight reduction under laser irradiation, illustrating the ultrafast hot electrons transfer (Fig. 2c). However, the radii of the semicircle of M/CdS showed an obvious reduction under laser irradiation (Fig. 2d), suggesting the excellent charge transfer with the temperature variation. To investigate the advantages of composite, the EIS Nyquist plots of CdS, MXene, and M/CdS under laser irradiation were further studied. As presented in Fig. 2e, the radii of semicircle for M/CdS possessed a smaller radius than CdS, which 
revealed that MXene significantly accelerated the separation of the pyroelectric-induced electric charges and resulted in a reduced resistance.

Then, the pyroelectric current and potential were further studied. By means of the COMSOL finite element simulation, the simulated pyro-potential distribution across CdS was characterized, as shown in Fig. $2 \mathrm{f}$, in which the pyro-potential changes from 0 to $11.8 \mathrm{mV}$ with a temperature variation of $50^{\circ} \mathrm{C}$. Subsequently, the photothermal effect, pyroelectric current, and potential response of CdS and M/CdS under 1064-nm laser irradiation were measured. As presented in Fig. $2 \mathrm{~g}$, MXene and M/CdS exhibited a significant rise in temperature $\left(\Delta \mathrm{T}=45^{\circ} \mathrm{C}\right.$ and $\left.52^{\circ} \mathrm{C}\right)$ for $10 \mathrm{~min}$ than $\mathrm{CdS}$ alone. Similarly, the infrared thermal image (ITI) visually proved this photothermal effect of $M / C d S$ as well (Fig. $2 \mathrm{~h}$ ). The excellent photothermal performance of M/CdS could be applied in PTT and pyroelectric catalysis. According to Fig. 2i, j, neither pyroelectric current nor potential variation of CdS was detected. The reason is that the pyroelectric field could not be formed in CdS upon low-energy 1064-nm laser irradiation. On the contrary, with the addition of MXene, M/CdS showed a significant pyroelectric current ( $20 \mathrm{nA})$ and potential (50 mV), which confirmed the pyroelectric effect of the complexed material under 1064-nm laser irradiation.

Finally, Mott-Schottky curves were studied for the type and flat-band potentials of MXene and CdS (Fig. 2k, I). The positive slope of CdS implied the characteristic of n-type semiconductor. The flat-band potentials of MXene and CdS were $-1.02 \mathrm{~V}$ and $-1.08 \mathrm{~V}$ vs. standard calomel electrode (SCE), respectively, which corresponded to $-0.775 \mathrm{~V}$ and $-0.835 \mathrm{~V}$ vs. normal hydrogen electrode (NHE, $\mathrm{pH} 7)$. The conductive band (CB) of CdS was calculated to be $-0.935 \mathrm{~V}$ because the $\mathrm{CB}$ of $n$-type semiconductors is always $0 \sim 0.1 \mathrm{~V}$ more negative than the flat-band potential ${ }^{33}$. Therefore, the CB potential of $\mathrm{CdS}$ is more negative than the redox potential of $\mathrm{O}_{2} / \cdot 0-2(-0.54 \mathrm{~V}$ vs. NHE, $\mathrm{pH} 7)$, which could accomplish the reduction of $\mathrm{O}_{2}$ to $\cdot 0-2$.

Pyroelectric catalysis. The mechanism of pyroelectric catalysis was shown in Fig. 3a. CdS and MXene formed a kind of Schottky heterojunction. Owing to the difference of Fermi level $\left(E_{f}\right)$ and work function between CdS and MXene, the negative charges of CdS transferred to MXene to equilibrate the $E_{f}$ between the two materials ${ }^{34}$. During the equilibrium process, the energy band of CdS (n-type semiconductor) bended upward to generate the Schottky barrier. MXene converted the light to hyperpyrexia with laser irradiation. Following the pyroelectric effect caused by temperature variation, the negative charges on the surface of CdS migrated along the Schottky junction to MXene. The Schottky barrier could prevent the backflow of negative charges and boost the separation of pyroelectric-excited positive charges and negative charges (Eq. 1) ${ }^{35}$. Therefore, MXene could be treated as a co-catalyst to enhance the catalytic efficiency of CdS. The isolated pyroelectric-excited negative charges can react with $\mathrm{O}_{2}$ to generate superoxide radicals $\left(\cdot \mathrm{O}_{2}^{-}\right)$and hydroxyl radical $(\cdot \mathrm{OH})$, while the associated positive charges can react with $\mathrm{H}_{2} \mathrm{O}$ to produce $\mathrm{O}_{2}$ and $\mathrm{H}^{+}$(Eqs. 2 and 3).

$M / C d S+\Delta T \rightarrow M / C d S\left(q^{+}+q^{-}\right),(1)$ 
$\mathrm{O}_{2}+\mathrm{q}^{-} \rightarrow \cdot \mathrm{O}-2 \rightarrow \cdot \mathrm{OH},(2)$

$2 \mathrm{H}_{2} \mathrm{O}+4 \mathrm{q}^{+} \rightarrow \mathrm{O}_{2}+4 \mathrm{H}^{+},(3)$

First, the $\mathrm{O}_{2}$ generation of $\mathrm{M} / \mathrm{CdS}$ under 1064-nm laser irradiation was studied using a dissolved oxygen meter. After $5 \mathrm{~min}$ of 1064-nm laser irradiation, $\mathrm{M} / \mathrm{CdS}$ could produce a significant amount of $\mathrm{O}_{2}$ in contrast to MXene and CdS, which proved the occurrence of water splitting by pyroelectric catalysis (Fig. 3b). Then, the ROS generation of M/CdS under 1064-nm laser irradiation was detected using the 1, 3-diphenylisobenzofuran (DPBF) bleaching method (Fig. 3c). MXene or CdS alone could not produce ROS under 1064-nm laser irradiation. The reason is that the hot electrons generated by surface plasmon resonance of MXene exhibited an ultrafast lifetime, and CdS could not be excited by NIR-II laser (1064 $\mathrm{nm}, 1.17 \mathrm{eV}$ ) due to its wide energy gap. However, owing to the formulation of negative charges and positive charges for $\mathrm{CdS}$ by pyroelectric effect, the M/CdS could generate the ROS. Furthermore, we further verified that pyroelectric catalysis-based water splitting provided the $\mathrm{O}_{2}$ for ROS generation. We added $\mathrm{N}_{2}$ into the $\mathrm{M} / \mathrm{CdS}$ solution to remove the extra $\mathrm{O}_{2}$. The results indicated the M/CdS isolated with $\mathrm{O}_{2}$ could still produce ROS, which proved the M/CdS could decompose $\mathrm{H}_{2} \mathrm{O}$ to $\mathrm{O}_{2}$ for ROS generation. Besides, the M/CdS + lactic acid (LA) generated more ROS than M/CdS, which was reasoned that the LA as a sacrificial agent could react with positive charges (Eq. 4), leading to the decrease of charge recombination for enhanced catalysis ${ }^{27,36}$.

$\mathrm{LA}+\mathrm{q}^{+} \rightarrow \mathrm{CO}_{2}+\mathrm{H}_{2} \mathrm{O}$ (4)

Next, the pyroelectric catalysis-mediated water splitting of M/CdS was studied, as shown in Fig. 3d. We designed a visual experiment, which proved the water splitting through the decrease of solution volume. Under 2 min of 1064-nm laser irradiation, the MXene solution volume did not change, which indicated the evaporation and expansion by heat could be neglected. Accordingly, the M/CdS showed an apparent decrease in solution volume and gas generation. Thus, the result revealed the M/CdS could realize the water splitting under 1064-nm laser irradiation through pyroelectric catalysis. Subsequently, we used the Matrigel to simulate the ECM for the penetration of MXene and M/CdS. As shown in Fig. 3e, the M/CdS exhibited better penetration in contrast to MXene under 1064-nm laser irradiation and laser alone. The reason might be that the $\mathrm{M} / \mathrm{CdS}$ could produce heat and decompose water simultaneously, leading to excellent penetration.

The photothermal stability of M/CdS was tested as well. As presented in Fig. 3f, M/CdS could keep the immobile photothermal effect during three laser irradiation cycles, which was better than MXene alone. Furthermore, after 5 min of laser irradiation, the morphology of M/CdS showed no significant difference, suggesting its good photothermal stability (Supplementary Fig. 2). It is known that the NIR-II laser exhibits superior tissue penetration ${ }^{37-39}$. Thus, we covered a chicken breast $(5 \mathrm{~mm})$ on the samples to further measure the photothermal effect. According to Fig. 3g, the temperature rise presented no significant change after the cover of chicken breast, which indicated that the excellent tissue penetration 
of NIR-II (1064 nm) laser and potentials of M/CdS for further in vivo PTT. The above results proved that $\mathrm{M} / \mathrm{CdS}$ showed excellent photothermal stability, ROS generation, water splitting, and ECM penetration, which confirmed the pyroelectric-catalytic process we proposed.

Cellular uptake and cytotoxicity. The cellular uptake and cytotoxicity of M/CdS-HA were studied using HeLa and LO2 cells. To localize the position of M/CdS-HA in cells, the fluorescent rhodamine B was modified with HA to form M/CdS-HA/RB. As shown in Fig. 4a, the M/CdS-HA/RB exhibited better cellular uptake in HeLa cells than LO2 cells. The reason was that the surficial HA of M/CdS-HA/RB could combine with the CD44 receptor on HeLa cells to improve the cellular uptake ${ }^{40-42}$. Furthermore, quantificationally, the flow cytometry (FCM) analysis proved that the M/CdS-HA/RB could mostly be taken into HeLa cells (Fig. 4b). Subsequently, the ROS generation in vitro was measured by a 2', 7'-dichlorofluorescein diacetate (DCFH-DA) probe. Under 1064-nm laser irradiation, the HeLa cells treated with PBS, MXene, and CdS presented hardly any fluorescence. However, the M/CdS-HA group showed noticeable green fluorescence of 2', 7'-dichlorofluorescein (DCF), which testified the ROS generation during the pyroelectric catalysis (Fig. 4c).

The cytotoxicity of all tested groups (PBS, MXene, CdS, M/CdS, and M/CdS-HA with/without 1064-nm laser irradiation) to HeLa cells was tested using MTT assay (Fig. 4d). Under laser irradiation, the MXene exhibited significant cytotoxicity $(P<0.0001$, compared with the dark group), which indicated the damage to HeLa cells of PTT. However, the CdS with laser irradiation showed no significant difference $(P=0.0696$, compared with the dark group), which was reasoned that the CdS could not be excited by the 1064-nm laser. Both $M / C d S$ and $M / C d S-H A$ with laser irradiation presented excellent cytotoxicity $(P<0.0001$, compared with dark groups). Furthermore, the HA modification of M/CdS-HA enhanced the biocompatibility (in the dark, $P=0.0001$ ) and target (laser irradiation, $P=0.0195$ ) compared with $M / C d S$. Besides, the results of fluorescein diacetate (FDA)/propidium iodide (PI) staining (Fig. 4e) and cell apoptosis tested by FCM (Fig. 4f) proved the superior cytotoxicity of M/CdS-HA under 1064-nm laser irradiation. Eventually, we studied the cytotoxicity of M/CdS-HA with laser irradiation under different environments to further reveal the therapeutic mechanism. The significant difference of all the tested groups was compared with $M / C d S-H A+L(G 1)$. According to Fig. $4 \mathrm{~g}$, the addition of LA (10 mM) enhanced the cytotoxicity $(P=0.0057)$, which proved the consumption of positive charges by $L A$ to enhance the reduction of negative charges in the pyroelectric catalysis. The cover of $5 \mathrm{~mm}$ of chicken breast did not affect the cytotoxicity $(P=0.5047)$, which elucidated the excellent tissue penetration of the 1064-nm laser. The addition of $\mathrm{NH}_{4} \mathrm{Cl}$ into HeLa cells could make a neutral environment, which hindered the reaction of $\mathrm{H}^{+}$in cellular lysosome with $\cdot 0-2$ to form stronger $\cdot \mathrm{OH}$. However, the addition of $\mathrm{NH}_{4} \mathrm{Cl}$ solution did not influence the cytotoxicity $(P=0.4116)$, which testified the generation of $\mathrm{H}^{+}$in the $\mathrm{O}_{2}$ evolution by pyroelectric catalysis-based water splitting. Additionally, under a hypoxia environment, the cytotoxicity of $M / C d S+L$ decreased $(P=0.0106)$, which reasoned that the $\mathrm{O}_{2}$ in the tumor could still react with negative charges to form ROS. The above results revealed the excellent cellular uptake and cytotoxicity of M/CdS-HA under 1064-nm laser irradiation. 
Penetration and cytotoxicity to multicell spheroids (MCSs). HeLa cells were seeded into a culture dish with an ultra-low attachment surface to form MCSs. To study the relationship between diffusion and transcytosis, an endocytosis inhibitor genistein (Gen) was used to pre-treated with MCSs to prevent the passage of M/CdS across the cell monolayer (Fig. 5a). We first revealed the MCSs penetration of M/CdS$\mathrm{HA} / \mathrm{RB}$ with laser irradiation from Day 0 to Day 6 using fluorescent imaging. As shown in Fig. $5 \mathrm{~b}$, the blue fluorescence meant the cell nucleus, and the red fluorescence localized in the center of MCSs, which suggests the M/CdS-HA penetrated MCSs under laser irradiation on Day 0 . Following the laser irradiation on Day 2, Day 4, and Day 6, the MCSs collapsed stepwise, which indicated the excellent penetration and damage to MCSs. Furthermore, we researched the efficacy of different samples (MXene $+L, M / C d S$, $\mathrm{M} / \mathrm{CdS}+\mathrm{L}$, and $\mathrm{M} / \mathrm{CdS}+\mathrm{L}+\mathrm{Gen}$ ) to MCSs by bright imaging (Fig. $5 \mathrm{c}$ ). On Day 0 , the sizes of MCSs in all the tested samples were consistent. After 6-day treatment, the M/CdS could not inhibit the proliferation of MCSs. The MXene $+\mathrm{L}$ group showed slight ablation of MCSs, which suggested the hyperpyrexia alone improved the MCSs penetration limitedly. However, the M/CdS $+L$ exhibited complete disintegration of MCSs, which proved better cytotoxicity to MCSs resulting from the enhanced MCSs penetration through hyperpyrexia and TIP decrease. It was noted that the addition of Gen could not affect the damage to MCSs of M/CdS $+L$, which revealed that the enhanced diffusion by the combination of hyperpyrexia and decrease of TIP was the main approach of M/CdS delivery rather than transcytosis. The results of MCSs viability by MTT assay were consistent with bright images (Fig. $5 \mathrm{~d}$ ). The above results testified the superior penetration and cytotoxicity of M/CdS with laser irradiation for MCSs.

Antitumor efficacy in vivo. The antitumor efficacy of M/CdS-HA was evaluated using U14 tumor xenograft models. The mice treated with Saline, CdS-HA + L, MXene-HA $+\mathrm{L}, \mathrm{M} / \mathrm{CdS}-\mathrm{HA}$, and M/CdS-HA+L were tested for 14 days. Moreover, the groups of MXene-HA with intratumor (i.t.) injection $+L$ and $M / C d S-$ $\mathrm{HA}$ with intravenous (i.v.) injection $+\mathrm{L}$ were studied for the 14-day treatment and 16-day drug withdrawal to test the tumor recurrence (Fig. 6a). As presented in Fig. 6b and Supplementary Fig. 3, the proliferation of tumors treated with $\mathrm{M} / \mathrm{CdS}-\mathrm{HA}+\mathrm{L}$ was inhibited significantly $\left(\mathrm{V} / \mathrm{V}_{0}=0.75\right.$, inhibition ratio: $\left.93.75 \%\right)$ compared with other groups. Then, we studied the hematoxylin and eosin (H\&E) staining and immunohistochemical staining (Caspase-3 and Ki67) of tumors treated with Saline and M/CdS-HA + L (Fig. 6c). H\&E staining presented the loose tissue of M/CdS-HA + L compared with the Saline group, which indicated the enhanced tumor apoptosis. Furthermore, the tumor treated with $\mathrm{M} / \mathrm{CdS}-\mathrm{HA}+\mathrm{L}$ showed high-expressed caspase-3 and low-expressed Ki67 compared with the Saline group, which further proved the superior antitumor efficacy of M/CdS-HA $+\mathrm{L}$. Also, we tested the ROS generation and photothermal effect of $M / C d S-H A+L$ in the tumor region. According to Fig. $6 d$, for the $M / C d S-H A+L$, both FCM analysis and DCF-based fluorescent imaging (DCF-FI) of the tumor section proved the ROS generation due to the presence of green fluorescence of DCF. ITI suggested the apparent rise of temperature in the tumor region, which illustrated the excellent photothermal effect of M/CdS-HA in vivo.

It is necessary to evaluate the biosafety of M/CdS-HA in vivo to assure its further clinical and translational potentials. Several biochemical indexes were measured to assess the side effect of therapeutic agents. Alanine aminotransferase (ALT) and aspartate aminotransferase (AST) were used to 
assess the liver functions, and urea nitrogen (BUN) was used to assess the kidney functions. According to Fig. 6e, the $M / C d S-H A+L$ group exhibited no significant difference on functions of liver and kidney compared with the Saline group for the experimental mice. Additionally, none of tested groups showed weight loss for the treated mice (Fig. 6f). Furthermore, the M/CdS-HA $+\mathrm{L}$ group showed no obvious organ injury or inflammatory lesions in the major organs (liver, spleen, kidney, heart, and lung) by H\&E staining compared with the Saline group (Fig. $6 \mathrm{~g}$ and Supplementary Fig. 4). The above results indicated the superior antitumor efficacy and biosafety of $M / C d S-H A+L$ for further clinical transfer.

Tumor penetration in vivo. The hyperpyrexia and pyroelectric catalysis-based decomposition of tumor interstitial fluid by M/CdS-HA + L could decrease the TIP, which enhances the tumor penetration of nanomedicine and blood perfusion. The enhanced blood perfusion could bring more nanomedicine to the tumor site, leading to a circle of improved drug delivery. Moreover, the over-expressed LA in the tumor could serve as a sacrificial agent to consume the positive charges generated in pyroelectric catalysis for inhibiting the charge recombination, which promoted the negative charge-based reduction for the enhancement of ROS generation. Therefore, the LA in the tumor microenvironment enabled the M/CdSHA to exhibit enhanced catalytic performance and antitumor efficacy under laser irradiation (Fig. 7a). First, we measured the changes of TIP in $120 \mathrm{~min}$. The TIP of the Saline group ( $38.2 \mathrm{mmHg}$ ) was identified as $100 \%$. According to Fig. $7 \mathrm{~b}$, the M/CdS-HA without laser irradiation could not decrease the TIP. The photothermal effect alone (MXene-HA $+\mathrm{L})$ decreased $18 \%$ of TIP $(31.3 \mathrm{mmHg})$, which indicated the hyperpyrexia straightforwardly melted the ECM and disrupted the fibroblasts. Unfortunately, the photothermal effect alone could not decrease the TIFP. The M/CdS-HA $+\mathrm{L}$ with both photothermal effect and pyroelectric catalysis-based decomposition of tumor interstitial fluid decreased $52 \%$ of TIP (18.3 $\mathrm{mmHg}$ ) at $100 \mathrm{~min}$. The results proved that the M/CdS-HA could sharply decrease the TIP under laser irradiation. Then, we measured the amount of LA in the tumor. As presented in Fig. 7c, the amounts of LA in tumors treated with MXene-HA $+\mathrm{L}$ and $M / C d S-H A$ had no significant difference compared with the Saline group. Nevertheless, the M/CdS-HA $+\mathrm{L}$ showed an evident decrease of $L A$ in tumor compared with the M/CdS-HA ( $P=0.0001)$. Therefore, the consumption of LA in tumor implied that the LA reacted with positive charges generated in pyroelectric catalysis to promote the negative charge-based reduction for enhanced ROS generation.

Because of the decrease of TIP, blood perfusion was improved, leading to more accumulation of nanomedicine in the tumor. We detected the blood perfusion in the same tumor region without and with 2 min of 1064-nm laser irradiation at different time points ( $0 \mathrm{~min}, 15 \mathrm{~min}$, and $30 \mathrm{~min}$ ). According to Fig. $7 \mathrm{~d}$, after 24-h i.v. injection of M/CdS-HA, the blood perfusion was very weak without laser irradiation. After 2 min of 1064-nm laser irradiation, the blood perfusion enhanced gradually with time. It was reasoned that the reduced TIP could rapidly regulate the pressure difference between the blood and tumor interstitial fluid, which provided a strong driving force for drug delivery from blood to the tumor. The regions pointed by the white arrows were the bleeding spots, which were formed by the increased blood flow and damage by hyperpyrexia. Simultaneously, the enhancement of blood perfusion mediated by M/CdS-HA + L could increase the intratumoral $\mathrm{O}_{2}$ content, which improved the therapeutic effect of "Hydrodynamic therapy". 
As shown in Fig. 7e, the Saline group exhibited hypoxia and no significant change under laser irradiation. In contrast, after the treatment of $\mathrm{M} / \mathrm{CdS}-\mathrm{HA}+\mathrm{L}$, the $\mathrm{O}_{2}$ content increased obviously, which attributed to the enhanced blood perfusion. The experiments provided forceful evidence for the improvement of the drug delivery following the enhanced blood perfusion.

Finally, we explored the tumor recurrence and tumor apoptosis of all tested groups. In the experiment of tumor recurrence, we chose MXene-HA with i.t. injection $+L$ as control. As revealed in Fig. $7 f$, due to the more accumulation of MXene-HA (i.t.) into tumors, the MXene-HA (i.t.) + L exhibited better tumor inhibition $\left(V / V_{0}=0.5\right)$ compared with $M / C d S-H A+L\left(V / V_{0}=0.75\right)$ with 14-day treatment. However, with 16-day drug withdrawal after treatment, the $M / C d S-H A+L$ indicated better inhibition to tumor recurrence $\left(V / V_{0}=1.2\right)$ compared with $M X e n e-H A$ (i.t. $)+L\left(V / V_{0}=3.0\right)$. The reason was that the $M / C d S-H A+L$ could damage the deep tumor stem cells, resulting from the excellent tumor penetration by TIFP decrease. Correspondingly, the MXene-HA (i.t.) + L could only damage the surficial tumor cells. Then, the cell apoptosis and Ki67 staining for the center of the tumor were studied, as shown in Fig. 7g. According to the FCM analysis, the $M / C d S-H A+L$ showed obvious cell apoptosis (97.45\%) compared with Saline (0.2\%), M/CdS-HA (1.0\%), $M X e n e-H A+L(65.6 \%)$ and MXene-HA (i.t.) $+L(77.7 \%)$. Consistently, the center of tumor treated with $M / C d S-H A+L$ exhibited the minimum expression of Ki67 compared with other tested groups, which proved the anabatic apoptosis. Taken together, the above results proved that the "Nano-lymphatic" exhibited the enhanced tumor penetration and inhibition of tumor recurrence.

\section{Discussion}

Although nanomedicines have brought great hope to tumor treatments in recent years, the inefficient penetration into tumor severely limits their application. Previous studies focused on size-shrinking of drugs $^{43-45}$, remodeling of tumor microenvironment ${ }^{46}$, and transcytosis ${ }^{47-49}$ to enhance the tumor penetration. However, none of these strategies overcome the key hinder of drug delivery: TIP, especially TIFP. Therefore, for the first time, we utilize pyroelectric catalysis-based water splitting to reduce TIFP and hyperpyrexia to reduce the TISP. Because this work simulates the lymphatic function to enhance the blood perfusion, we identify it as "Nano-lymphatic". Furthermore, the hyperpyrexia and ROS generated by NIR-II laser irradiation can efficiently damage the tumor cells. Benefit from the decrease of TIP, the "Nanolymphatic" exhibits enhanced tumor penetration and inhibition of tumor proliferation and recurrence.

In this contribution, the n-type semiconductor CdS and metalloid MXene are constructed to form a Schottky junction to realize further catalysis. Due to its wide energy gap, CdS cannot be excited by NIR-II light (1064 nm, $1.17 \mathrm{eV})$. Accordingly, we harness the pyroelectric effect of CdS under temperature variation to achieve the water splitting. The temperature variation can be realized through the photothermal effect of MXene under NIR-II light. However, the pyroelectric effect is hard to characterize. We utilize the COMSOL finite element simulation and electrochemical methods to prove the pyroelectric currents and potentials. Furthermore, we validated the photothermal effect, photothermal stability, water splitting, $\mathrm{O}_{2}$ evolution, and ROS generation of "Nano-lymphatic". 
We proved the superior antitumor effect of "Nano-lymphatic" by in vitro and in vivo experiments. Furthermore, the simulated tumor ECM by Matrigel, 3D tumor spheroids, and solid tumor born on the mice were used to prove the enhanced penetration of "Nano-lymphatic". The combination of hyperpyrexia and decomposition of tumor interstitial fluid reduces the TIP significantly. Thereinto, the TIFP is the major constituent of TIP. In addition, the "Nano-lymphatic" can decrease the amount of LA in tumors, which indicates that the LA is the sacrificial agent to react with positive charges, inhibiting the recombination of charge carriers for enhanced ROS generation. Based on the above results, we identify the tumorpenetrating therapeutic strategy based on water splitting and ROS generation as "Hydrodynamic therapy".

In summary, we designed a pyroelectric catalysis-based "Nano-lymphatic" to reduce TIP for enhanced tumor penetration and treatments. We first identify the "Nano-lymphatic" and "Hydrodynamic therapy" in this work. "Nano-lymphatic" represents a kind of nanomedicine to reduce the TIFP, and "Hydrodynamic therapy" is a newly emerging deep-penetrating therapeutic strategy with catalytic water splitting and ROS generation. Furthermore, we bring the pyroelectric catalysis into nanotechnology-based tumor treatments. It is expected that such concepts can be beneficial for future advances in drug delivery, catalytic nanomedicine, and cancer therapy.

\section{Methods}

Chemicals. Multi-layer $\mathrm{Nb}_{2} \mathrm{CT}_{\mathrm{x}}$ (MXene) was purchased from Jilin Yiyi Technology Co. Ltd. (Jilin, China). $\mathrm{Cd}\left(\mathrm{NO}_{3}\right)_{2} \cdot 4 \mathrm{H}_{2} \mathrm{O},\left(\mathrm{NaPO}_{3}\right)_{6}, \mathrm{Na}_{2} \mathrm{~S}, \mathrm{Gen}, \mathrm{DPBF}$ and $\mathrm{HA}$ were obtained from Aladdin Industrial Co. Ltd. (Shanghai, China). Rhodamine B was purchased from Tianjin Kaitong Chemical Agents Technology Co. Ltd. (Tianjin, China). $\mathrm{Na}_{2} \mathrm{SO}_{4}$ and LA were obtained from Tianjin Fengchuan Chemical Agents Technology Co. Ltd. (Tianjin, China). Hoechst 33342 was purchased from Beijing Solarbio Technology Co. Ltd. (Beijing, China). MTT and DCFH-DA were purchased from Shanghai Beyotime Biological Technology Co. Ltd. (Shanghai, China). The ELISA kits of AST, ALT and BUN were purchased from Nanjing Jiancheng Bioengineering Institute Co. Ltd. (Nanjing, China), and LA was purchased from Shanghai Jining Shiye Co. Ltd. (Shanghai, China). All chemical agents used were of analytical grade.

Cell line and animal model. The HeLa cells and LO2 cells were purchased from Shanghai Tianjing Biological Technology Co. Ltd. (Shanghai, China), which were cultured in Dulbecco's modified Eagle's medium containing $10 \%$ fetal bovine serum and $1 \%$ penicillin/streptomycin at $37^{\circ} \mathrm{C}$ containing $5 \% \mathrm{CO}_{2}$. U14 cells were purchased from the Chinese Academy of Medical Sciences Tumor Cell Bank (Beijing, China). A total of $500 \mathrm{HeLa}$ cells were seeded onto the ultralow attachment surface of a 24-well plate (Corning, USA) to obtain 3D multicellular spheroids. Female Kunming mice aged 5-7 weeks were purchased from Vital River Laboratory Animal Center (Beijing, China). The mice acclimated to standard laboratory environment for a week and were provided with equal food and water and maintained at $25^{\circ} \mathrm{C}$ with a relative humidity of $55 \%$. 
Synthesis of M/CdS-HA. First, 20 mg multi-layer MXene was dissolved into $20 \mathrm{~mL}$ ultrapure water. After the ultrasonic stripping, the single-layer MXene was prepared. Then, $15.4 \mathrm{mg} \mathrm{Cd}\left(\mathrm{NO}_{3}\right)_{2} \bullet 4 \mathrm{H}_{2} \mathrm{O}$ and 15.3 $\mathrm{mg}\left(\mathrm{NaPO}_{3}\right)_{6}$ were dissolved into $29 \mathrm{~mL}$ ultrapure water, which added into single-layer MXene solution with one-hour stirring. Then, $1 \mathrm{~mL} \mathrm{Na} \mathrm{N}_{2} \mathrm{~S}$ solution $(0.4 \mathrm{mg} / \mathrm{mL})$ was rapidly added into above solution. The color of the system converted from black to dark green. The M/CdS was synthesized after a half-hour stirring. Finally, the precipitation of M/CdS after centrifugation was resuspended and incubated with 1 $\mathrm{mg} / \mathrm{mL}$ of HA solution for 2 hours. The M/CdS-HA was obtained after centrifugal purification.

Characterization of structure and performance. TEM (HT7700, Japan) was used for characterization of the M/CdS. The samples were prepared onto a copper grid coated carbon film for TEM measurement. DLS (Malvern Zetasizer Nano ZS90, UK) was used to measure the mean diameters of MXene, CdS, $\mathrm{M} / \mathrm{CdS}$ and M/CdS-HA. AFM (Bruker AXS Inc, USA) in tapping-mode was used to probe the morphology, size and height of the M/CdS. To determine the photothermal effect, $500 \mu \mathrm{L}$ of $\mathrm{M} / \mathrm{CdS}$ was placed in a $1.5 \mathrm{~mL}$ Eppendorf tube and exposed to a 1064-nm laser (1.0 W/cm², LSR-1064H-2W-19031303C, China) for 10 min. Dissolved Oxygen Meter (AZ Instrument Corp.) was used to test the $\mathrm{O}_{2}$ generation. To determine the ROS production, DPBF (absorbance at $410 \mathrm{~nm}$ ) bleaching method was performed. Water splitting experiment was explored using glass capillary (inner diameter: $0.9 \sim 1.1 \mathrm{~mm}$, Instrument Factory of West China Medical University, China). Stimulated ECM penetration of MXene and M/CdS under 1064$\mathrm{nm}$ laser irradiation (2 $\mathrm{min}$ ) was tested by Matrigel.

Pyro-electrochemical measurements. The time-dependent pyroelectric current and potential, electrochemical impedance spectroscopy Nyquist plots and Mott-Schottky curves were recorded on a CHI660e-type electrochemical workstation (Shanghai, China) connected with a standard three-electrode system (Calomel reference electrode and graphite counter electrode) placed in the electrolytic cell. The fluorine-doped tin oxide glass was selected as the working electrode. The electrolyte solution was $\mathrm{Na}_{2} \mathrm{SO}_{4}$ solution $(0.1 \mathrm{M})$. The samples (MXene, $\mathrm{CdS}$ and $\mathrm{M} / \mathrm{CdS}$ ) were deposited on the working electrode by an electrophoresis system. Thereafter, the working electrode was dipped into the electrolyte solution. Finally, the working electrode was stimulated under 1064-nm laser irradiation for the measurement of electrochemical impedance, pyroelectric current and potential. The pyro-potential distribution across the CdS was stimulated with the aid of the COMSOL Multiphysics.

Cellular uptake and cytotoxicity. The fluorescent rhodamine B was modified with M/CdS-HA to form M/CdS-HA/RB for cellular imaging. The cellular uptake of M/CdS-HA/RB to HeLa and LO2 cells was observed by inverted fluorescence microscope and flow cytometer (FCM, BD FACSCablibur, USA). The cell nucleuses were stained by Hoechst 33342. The cytotoxicity of all tested samples was studied by HeLa cells using the MTT assay. The following formula was used to calculate the viability of cell growth: Cell viability $(\%)=($ mean of absorbance value of treatment group / mean of absorbance value of control $) \times$ 100. The live and dead cells were stained by FDA/PI and Annexin V-FITC/PI for imaging and cell apoptosis. The cellular ROS was detected by DCFH. The non-fluorescent DCFH converted to DCF with green fluorescence under the ROS. 
Penetration and cytotoxicity to MCSs. The cell nucleuses of MCSs were stained by Hoechst 33342 . The M/CdS-HA/RB was added into the MCSs under 1064-nm laser irradiation on Day 0, Day 2, Day 4 and Day 6. The fluorescent images of MCSs were taken on Day 0, Day 2, Day 4 and Day 6 . The MXene $+L, M / C d S$, $\mathrm{M} / \mathrm{CdS}+\mathrm{L}$ and $\mathrm{M} / \mathrm{CdS}+\mathrm{L}+\mathrm{Gen}$ were added into the $300 \mu \mathrm{m}$ of MCSs for deep penetration in vitro. The bright images of MCSs were taken on Day 0 and Day 6 . The viability of the MCSs was measured by MTT assay.

Antitumor effect and biosafety in vivo. Kunming mice were inoculated subcutaneously with U14 cells. The mice were injected with $200 \mu \mathrm{L}$ of all the samples on the Day - 1 and Day 7 and irradiated by 1064$\mathrm{nm}$ laser on the Day 0 and Day 8 for the 14-day treatment. The tumor sizes were measured using a ruler during the experiment, and the volumes of tumors were calculated according to the formula: tumor volume $=$ length $\times(\text { width })^{2} / 2$. On the Day 14 , the mice were sacrificed under ether anesthesia and the tumors were removed, weighed, and photographed. The ROS generation in vivo was measured using DCF with green fluorescence by FCM and fluorescent section. The change of temperature in tumor region was measured using infrared thermal camera. The tumor and the main organs of the mice were examined by histological analysis (H\&E, Caspase-3 and Ki67). The serum separated from the ocular blood was used to assess the biochemical factors (AST, ALT and BUN). The weights of mice were measured every other day. The tumor recurrence was analyzed for the 16-day drug withdrawal and measured on the Day 30 . The dose of CdS used in vivo was $4.08 \mathrm{mg} / \mathrm{kg}$, which was much less than the lethal dosage of $\mathrm{Cd}(50 \mathrm{mg} / \mathrm{kg})$. The low dose of CdS, enhanced tumor target by loading on MXene and modification of HA led to the excellent biosafety of CdS in vivo.

Tumor penetration in vivo. The TIP level was measured by the WIN technique. Briefly, the pressure detector was calibrated before measurements. The needle was attached to a pressure transducer via a PE-50 polyethylene tube filled with sterile heparinized saline. The anesthetized mice, whose body temperature was maintained at $37.5^{\circ} \mathrm{C}$ with a heating pad, were carefully unhaired above the tumor. The needle was inserted into the central area of the tumor to test the TIP. The amount of LA in tumor was tested by ELISA kit. The tumor-bearing mice injected with Saline and M/CdS-HA were anesthetized, and the photoacoustic imaging system and blood oxygen imaging system were used to detect blood perfusion and blood oxygen concentration without and with 2 min of 1064-nm laser irradiation at different time points ( 0 min, $15 \mathrm{~min}$ and $30 \mathrm{~min}$ ). The cell apoptosis of central tumor was tested by FCM and Ki67 staining section.

\section{Ethics statement.}

All animal procedures were performed in accordance with the statute of laboratory animal ethics committee of Yanshan University to ensure the low suffering for the animals, which were approved by the Animal Ethics Committee of Yanshan University.

Statistical analysis. The statistical analysis was performed by using Student's two-tailed t-test with statistical significance assigned at $P<0.05$ (significant), $P<0.01$ (moderately significant), and $P<0.001$ 
(highly significant). All statistical analyses were performed by GraphPad Prism 8.0 and SPSS 20.0.

\section{Declarations}

\section{Data Availability}

Data supporting the findings of this study are available within the paper and its Supplementary Information files. The data that support the findings of this study are available from the corresponding author on reasonable request. Source data are provided with this paper.

\section{Competing interests}

The authors declare no competing interests.

\section{Author contributions}

Y. H., C. C. and D. G. designed the research. Y. H., Z. L., F. Y., K. Z., Y. Y., Y. L., L. Z. and Z. M performed the experiments in the research. Y. H. and D. G. wrote the paper. J. Y. and X. Z. revised the paper.

\section{Acknowledgements}

This work was supported by the National Natural Science Foundation of China (Nos. 22078280, 21776238 and 31801198), the Natural Science Foundation of Hebei Province (No. B2019203479), the Department of Education of Hebei Province (No. ZD2017084), and the Youth Program of National Nature Science Foundation of China (No. 22006128).

\section{References}

1. Zhou, Q. et al. Enzyme-activatable polymer-drug conjugate augments tumour penetration and treatment efficacy. Nat. Nanotechnol. 14, 799-809 (2019).

2. Poon, W., Kingston, B. R., Ouyang, B., Ngo, W. \& Chan, W. C. W. A framework for designing delivery systems. Nat. Nanotechnol. 15, 819-829 (2020).

3. Izci, M., Maksoudian, C., Manshian, B. B. \& Soenen, S. J. The Use of Alternative Strategies for Enhanced Nanoparticle Delivery to Solid Tumors. Chem. Rev. acs.chemrev.0c00779 (2021).

4. Lee, S. H. et al. Deep Tumor Penetration of Drug-Loaded Nanoparticles by Click Reaction-Assisted Immune Cell Targeting Strategy. J. Am. Chem. Soc. 141, 13829-13840 (2019).

5. Wang, Z. et al. Near-infrared light and tumor microenvironment dual responsive size-switchable nanocapsules for multimodal tumor theranostics. Nat Commun 10, 4418 (2019).

6. Heldin, C.-H., Rubin, K., Pietras, K. \& Östman, A. High interstitial fluid pressure-an obstacle in cancer therapy. Nat Rev Cancer 4, 806-813 (2004).

7. Gao, X. et al. Reducing Interstitial Fluid Pressure and Inhibiting Pulmonary Metastasis of Breast Cancer by Gelatin Modified Cationic Lipid Nanoparticles. ACS Appl. Mater. Interfaces 9, 29457- 
29468 (2017).

8. Rofstad, E. K., Galappathi, K. \& Mathiesen, B. S. Tumor Interstitial Fluid Pressure-A Link between Tumor Hypoxia, Microvascular Density, and Lymph Node Metastasis. Neoplasia 16, 586-594 (2014).

9. Stapleton, S., Milosevic, M., Tannock, I. F., Allen, C. \& Jaffray, D. A. The intra-tumoral relationship between microcirculation, interstitial fluid pressure and liposome accumulation. Journal of Controlled Release 211, 163-170 (2015).

10. Teng, P., Hood, B. L., Sun, M., Dhir, R. \& Conrads, T. P. Differential Proteomic Analysis of Renal Cell Carcinoma Tissue Interstitial Fluid. J. Proteome Res. 10, 1333-1342 (2011).

11. Provenzano, P. P. et al. Enzymatic Targeting of the Stroma Ablates Physical Barriers to Treatment of Pancreatic Ductal Adenocarcinoma. Cancer Cell 21, 418-429 (2012).

12. Milosevic, M., Fyles, A., Hedley, D. \& Hill, R. The human tumor microenvironment: invasive (needle) measurement of oxygen and interstitial fluid pressure. Seminars in Radiation Oncology 14, 249-258 (2004).

13. Ding, J. et al. Engineered nanomedicines with enhanced tumor penetration. Nano Today 29, 100800 (2019).

14. Gong, H. et al. Hyaluronidase To Enhance Nanoparticle-Based Photodynamic Tumor Therapy. Nano Lett. 16, 2512-2521 (2016).

15. Zhou, H. et al. Hyaluronidase Embedded in Nanocarrier PEG Shell for Enhanced Tumor Penetration and Highly Efficient Antitumor Efficacy. Nano Lett. 16, 3268-3277 (2016).

16. Agarwal, P. et al. Microfluidics Enabled Bottom-Up Engineering of 3D Vascularized Tumor for Drug Discovery. ACS Nano 11, 6691-6702 (2017).

17. Ji, T. et al. Designing Liposomes To Suppress Extracellular Matrix Expression To Enhance Drug Penetration and Pancreatic Tumor Therapy. ACS Nano 11, 8668-8678 (2017).

18. Stapleton, S. et al. Radiation and Heat Improve the Delivery and Efficacy of Nanotherapeutics by Modulating Intratumoral Fluid Dynamics. ACS Nano 12, 7583-7600 (2018).

19. Cong, C. et al. "Nano-lymphatic" photocatalytic water-splitting for relieving tumor interstitial fluid pressure and achieving hydrodynamic therapy. Mater. Horiz. 7, 3266-3274 (2020).

20. Cui, X. et al. Water-Splitting Based and Related Therapeutic Effects: Evolving Concepts, Progress, and Perspectives. Small 16, 2004551 (2020).

21. Wang, S. et al. Efficient photocatalytic overall water splitting on metal-free 1D SWCNT/2D ultrathin $\mathrm{C}_{3} \mathrm{~N}_{4}$ heterojunctions via novel non-resonant plasmonic effect. Applied Catalysis B: Environmental 278, 119312 (2020).

22. Wang, Q. \& Domen, K. Particulate Photocatalysts for Light-Driven Water Splitting: Mechanisms, Challenges, and Design Strategies. Chem. Rev. 120, 919-985 (2020).

23. Zhao, C., Chen, Z., Shi, R., Yang, X. \& Zhang, T. Recent Advances in Conjugated Polymers for VisibleLight-Driven Water Splitting. Adv. Mater. 32, 1907296 (2020). 
24. Xiao, L. et al. Pyroelectric nanoplates for reduction of $\mathrm{CO}_{2}$ to methanol driven by temperaturevariation. Nat Commun 12, 318 (2021).

25. Wang, C., Tian, N., Ma, T., Zhang, Y. \& Huang, H. Pyroelectric catalysis. Nano Energy 78, 105371 (2020).

26. You, H. et al. Room-temperature pyro-catalytic hydrogen generation of 2D few-layer black phosphorene under cold-hot alternation. Nat Commun 9, 2889 (2018).

27. Zhang, M., Hu, Q., Ma, K., Ding, Y. \& Li, C. Pyroelectric effect in CdS nanorods decorated with a molecular Co-catalyst for hydrogen evolution. Nano Energy 73, 104810 (2020).

28. Tang, Z. et al. Pyroelectric nanoplatform for NIR-II-triggered photothermal therapy with simultaneous pyroelectric dynamic therapy. Mater. Horiz. 5, 946-952 (2018).

29. Yu, Z., Zhou, P., Pan, W., Li, N. \& Tang, B. A biomimetic nanoreactor for synergistic chemiexcited photodynamic therapy and starvation therapy against tumor metastasis. Nat Commun 9, 5044 (2018).

30. Cheng, Y. et al. Spatiotemporally Synchronous Oxygen Self-Supply and Reactive Oxygen Species Production on Z-Scheme Heterostructures for Hypoxic Tumor Therapy. Adv. Mater. 32, 1908109 (2020).

31. Wu, J. et al. Strong pyro-catalysis of pyroelectric $\mathrm{BiFeO}_{3}$ nanoparticles under a room-temperature cold-hot alternation. Nanoscale 8, 7343-7350 (2016).

32. Wang, M., Wang, B., Huang, F. \& Lin, Z. Enabling PIEZOpotential in PIEZOelectric Semiconductors for Enhanced Catalytic Activities. Angew. Chem. Int. Ed. 58, 7526-7536 (2019).

33. Huang, X. et al. Insight into the piezo-photo coupling effect of $\mathrm{PbTiO}_{3} / \mathrm{CdS}$ composites for piezophotocatalytic hydrogen production. Applied Catalysis B: Environmental 282, 119586 (2021).

34. Zuo, G. et al. Ultrathin $\mathrm{ZnIn}_{2} \mathrm{~S}_{4}$ Nanosheets Anchored on $\mathrm{Ti}_{3} \mathrm{C}_{2} \mathrm{~T}_{X} \mathrm{MXene}$ for Photocatalytic $\mathrm{H}_{2}$ Evolution. Angew. Chem. 132, 11383-11388 (2020).

35. $\mathrm{Li}$, Y. et al. 2D/2D heterostructure of ultrathin $\mathrm{BiVO}_{4} / \mathrm{Ti}_{3} \mathrm{C}_{2}$ nanosheets for photocatalytic overall Water splitting. Applied Catalysis B: Environmental 285, 119855 (2021).

36. Waiskopf, N., Ben-Shahar, Y. \& Banin, U. Photocatalytic Hybrid Semiconductor-Metal Nanoparticles; from Synergistic Properties to Emerging Applications. Adv. Mater. 30, 1706697 (2018).

37. Chitgupi, U. et al. Surfactant-Stripped Micelles for NIR-II Photoacoustic Imaging through $12 \mathrm{~cm}$ of Breast Tissue and Whole Human Breasts. Adv. Mater. 31, 1902279 (2019).

38. Chen, Y.-S., Zhao, Y., Yoon, S. J., Gambhir, S. S. \& Emelianov, S. Miniature gold nanorods for photoacoustic molecular imaging in the second near-infrared optical window. Nat. Nanotechnol. 14, 465-472 (2019).

39. Chang, M. et al. A Multifunctional Cascade Bioreactor Based on Hollow-Structured $\mathrm{Cu}_{2} \mathrm{MoS}_{4}$ for Synergetic Cancer Chemo-Dynamic Therapy/Starvation Therapy/Phototherapy/Immunotherapy with Remarkably Enhanced Efficacy. Adv. Mater. 31, 1905271 (2019). 
40. Shen, S. et al. A nanotherapeutic strategy to overcome chemotherapeutic resistance of cancer stemlike cells. Nat. Nanotechnol. 16, 104-113 (2021).

41. Li, C. et al. Artificially Reprogrammed Macrophages as Tumor-Tropic Immunosuppression-Resistant Biologics to Realize Therapeutics Production and Immune Activation. Adv. Mater. 31, 1807211 (2019).

42. Meng, J. et al. A multistage assembly/disassembly strategy for tumor-targeted CO delivery. Sci. Adv. 6, eaba1362 (2020).

43. Wang, L. et al. Self-Reporting and Splitting Nanopomegranates Potentiate Deep Tissue Cancer Radiotherapy via Elevated Diffusion and Transcytosis. ACS Nano 14, 8459-8472 (2020).

44. Lin, X. et al. An Ultrasound Activated Vesicle of Janus Au-MnO Nanoparticles for Promoted Tumor Penetration and Sono-Chemodynamic Therapy of Orthotopic Liver Cancer. Angew. Chem. Int. Ed. 59, 1682-1688 (2020).

45. Cao, W. et al. NIR light triggered size variable "remote-controlled cluster bomb" for deep penetration and tumor therapy. Chemical Engineering Journa/ 375, 122080 (2019).

46. Li, H.-J. et al. Smart Superstructures with Ultrahigh pH-Sensitivity for Targeting Acidic Tumor Microenvironment: Instantaneous Size Switching and Improved Tumor Penetration. ACS Nano 10, 6753-6761 (2016).

47. Ju, C. et al. Sequential Intra-Intercellular Nanoparticle Delivery System for Deep Tumor Penetration. Angew. Chem. Int. Ed. 53, 6253-6258 (2014).

48. Sindhwani, S. et al. The entry of nanoparticles into solid tumours. Nat. Mater. 19, 566-575 (2020).

49. Liu, Y. et al. Transcytosis of Nanomedicine for Tumor Penetration. Nano Lett. 19, 8010-8020 (2019).

\section{Figures}


a
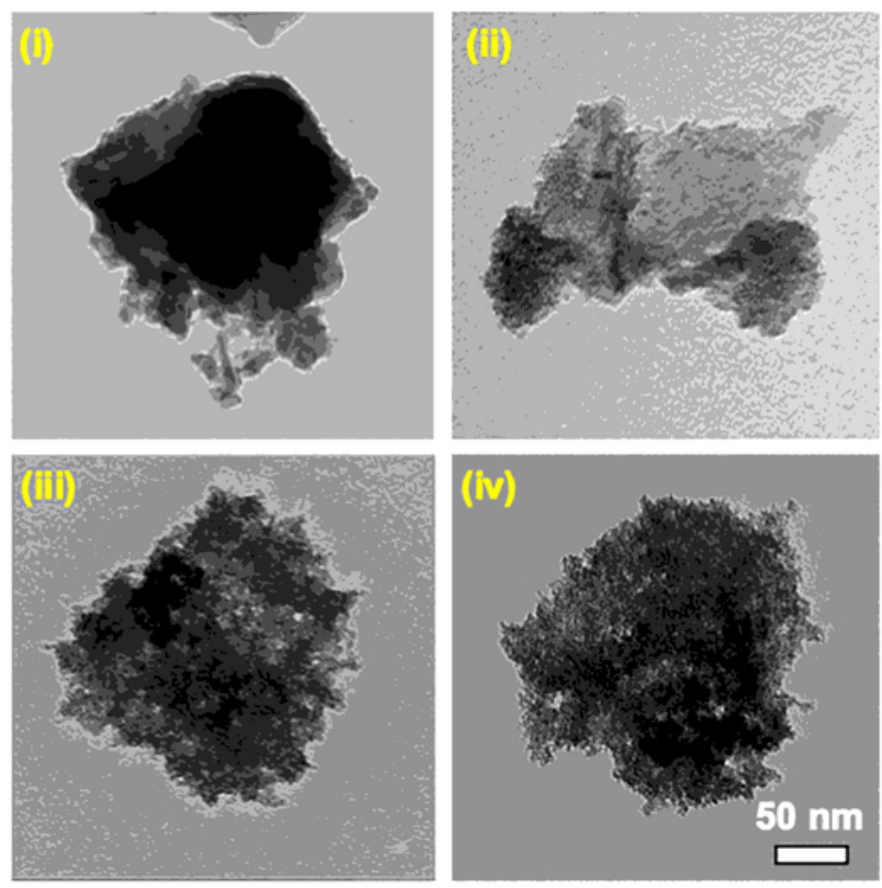

b

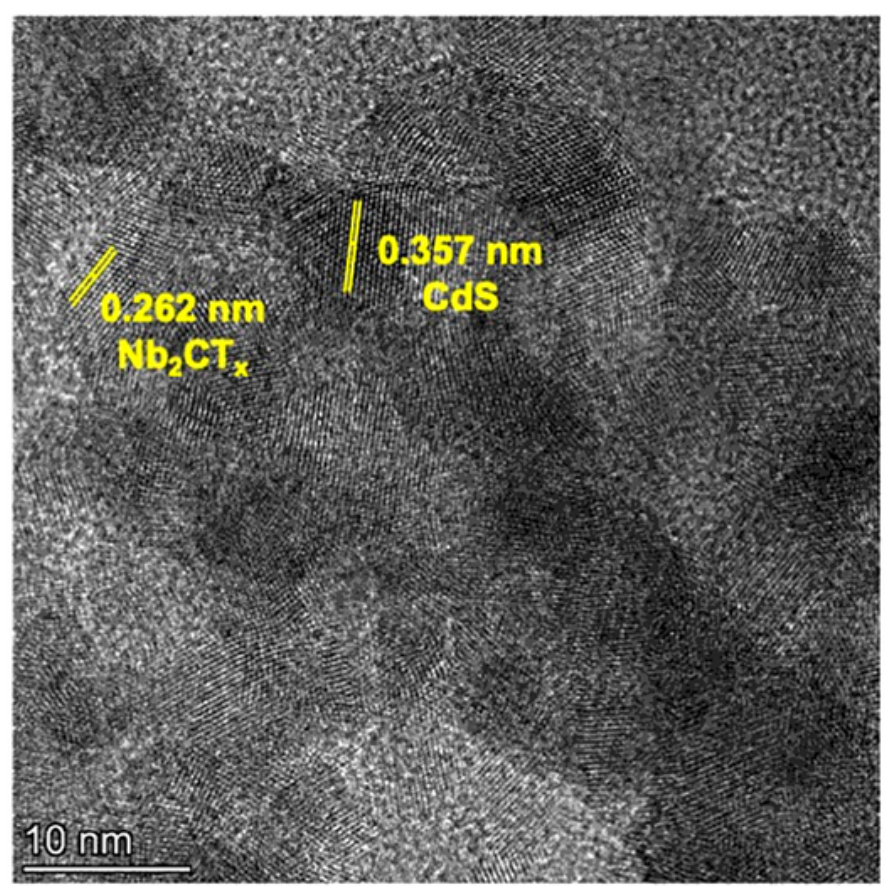

C

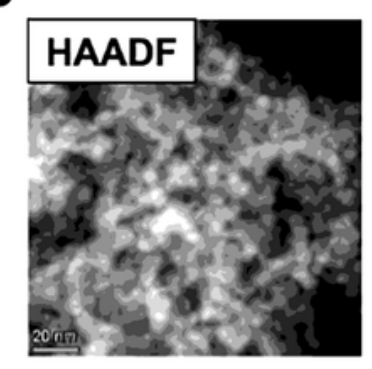

d

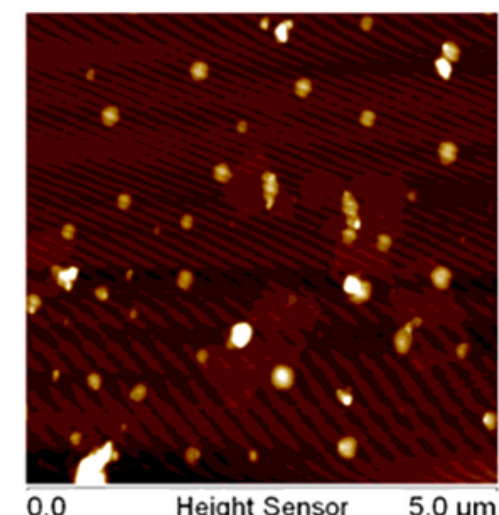

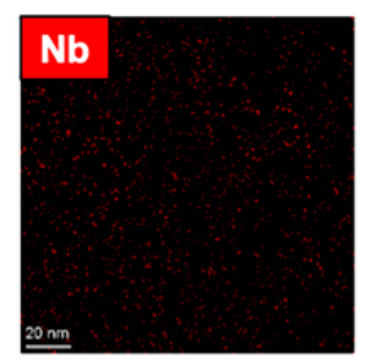
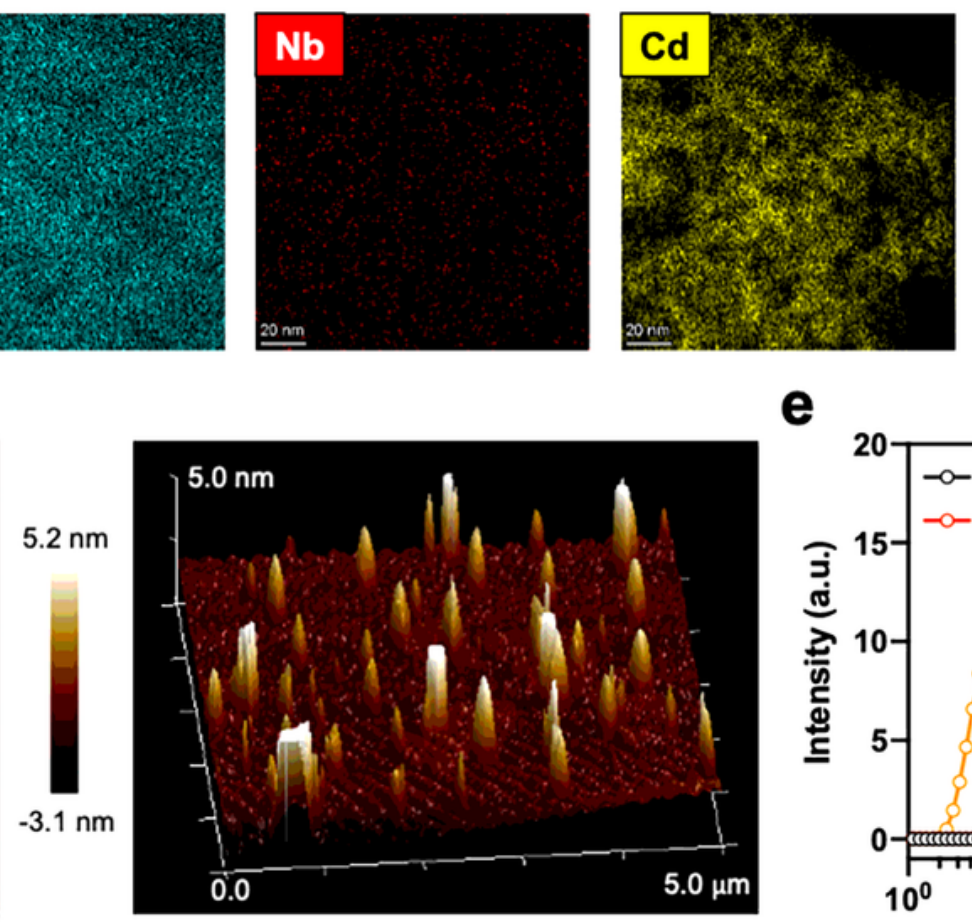

e

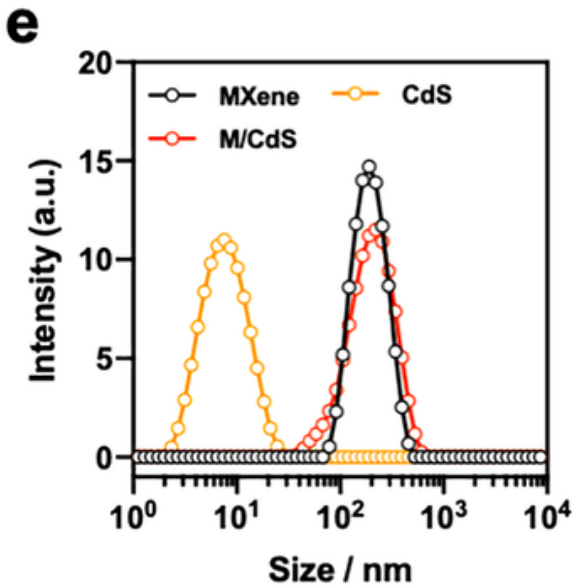

Figure 1

(a) TEM images of (i) multi-layer MXene, (ii) ultrathin MXene, (iii) M/CdS and (iv) M/CdS-HA . (b) HRTEM image of M/CdS. (c) STEM-HAADF image and elements mapping of M/CdS. (d) AFM image of M/CdS. (e) Size distribution of MXene, CdS, and M/CdS. 
a
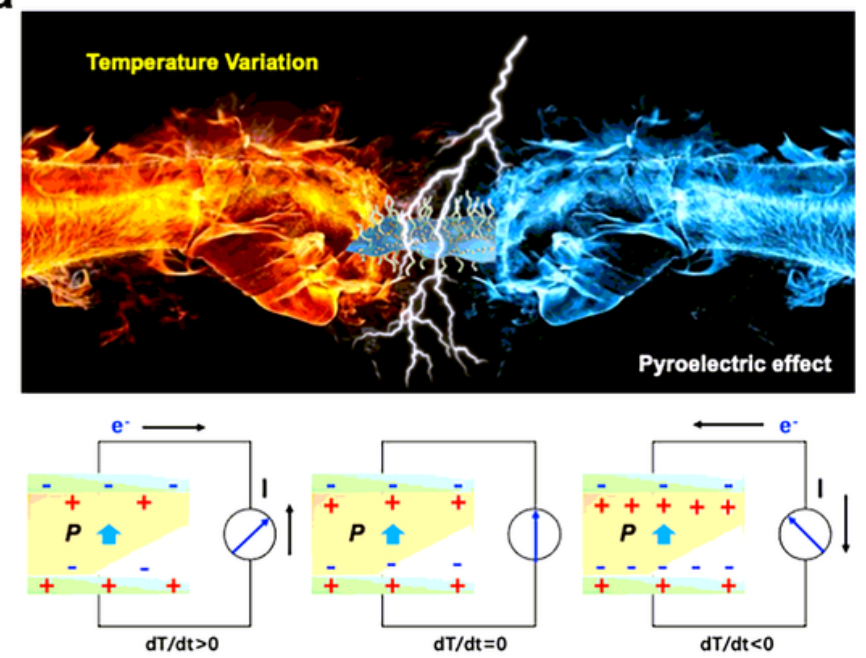

f

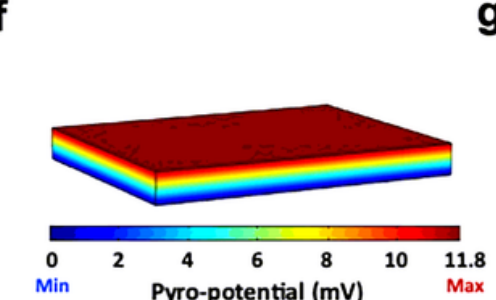

h

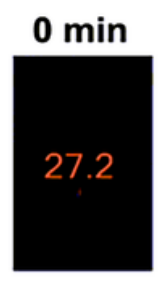

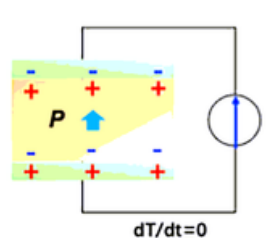

g

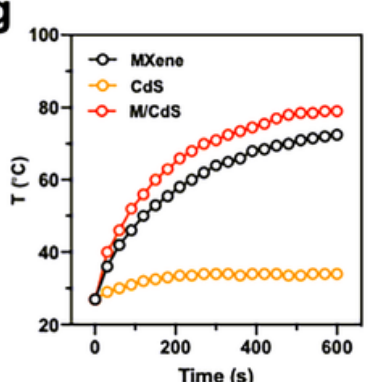

Time (s)
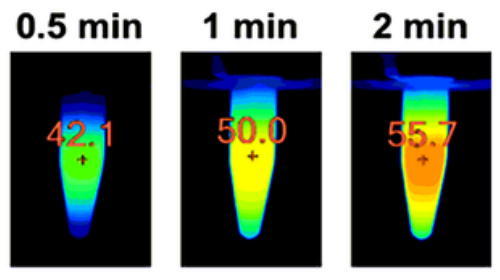

b
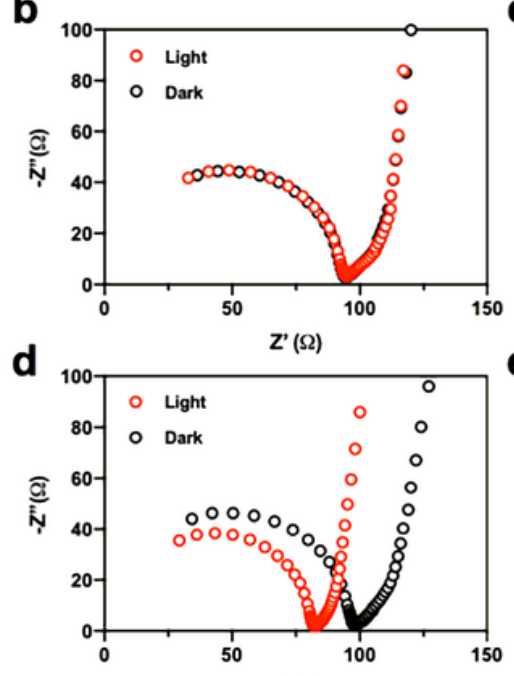

i

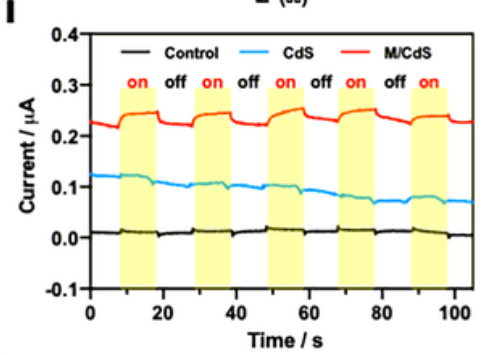

$\mathbf{k}$

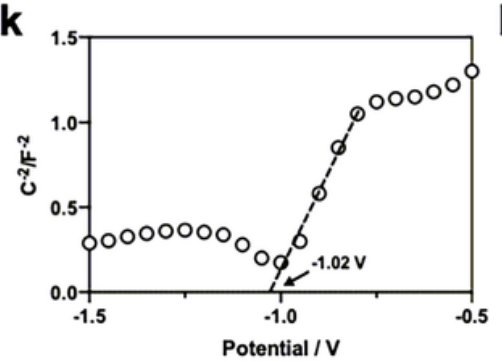

C
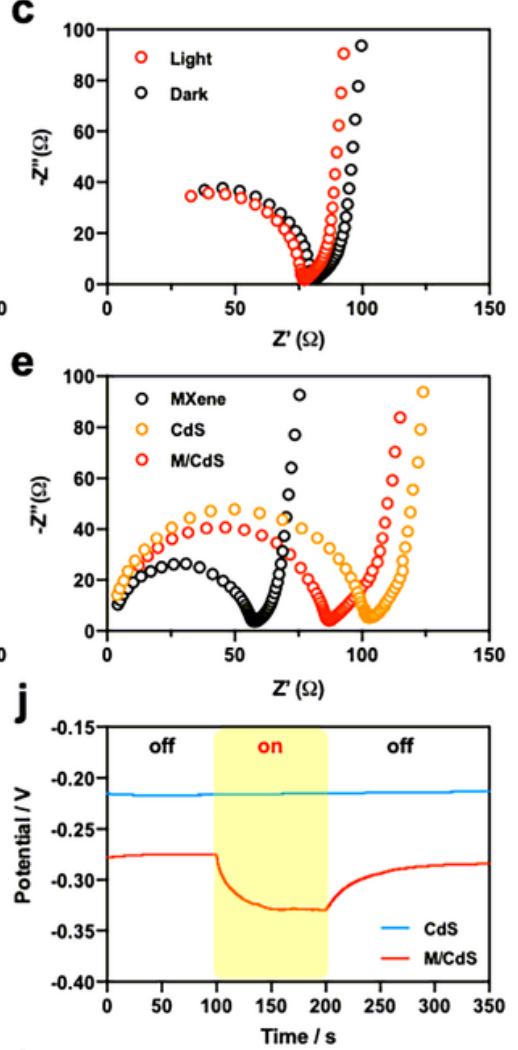

I

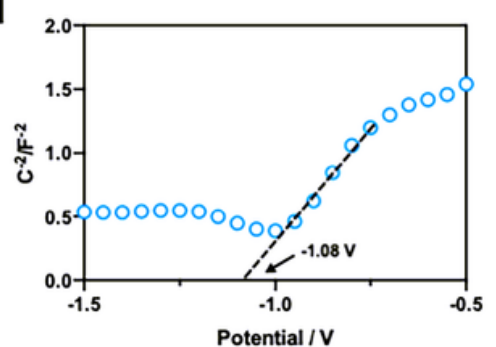

Figure 2

(a) Schematic illustration of pyroelectric effect and relationship between temperature variation (dT/dt) and pyroelectric current (I). EIS Nyquist plots of (b) CdS, (c) MXene and (d) M/CdS in dark and 1064-nm laser irradiation (1.0 W/cm2). (e) EIS Nyquist plots of CdS, MXene, and M/CdS under laser irradiation. (f) COMSOL finite element simulation of pyro-potential $\left(\Delta \mathrm{T}=50^{\circ} \mathrm{C}\right)$ for $\mathrm{CdS}$. (g) Photothermal effect of CdS, MXene, and M/CdS. (h) ITI of M/CdS under laser irradiation (3 min). (i) Pyroelectric currents of CdS and $\mathrm{M} / \mathrm{CdS}$ under laser irradiation. (j) Pyroelectric potentials of CdS and M/CdS under laser irradiation. MottSchottky curves of (k) MXene and (l) CdS. 
a

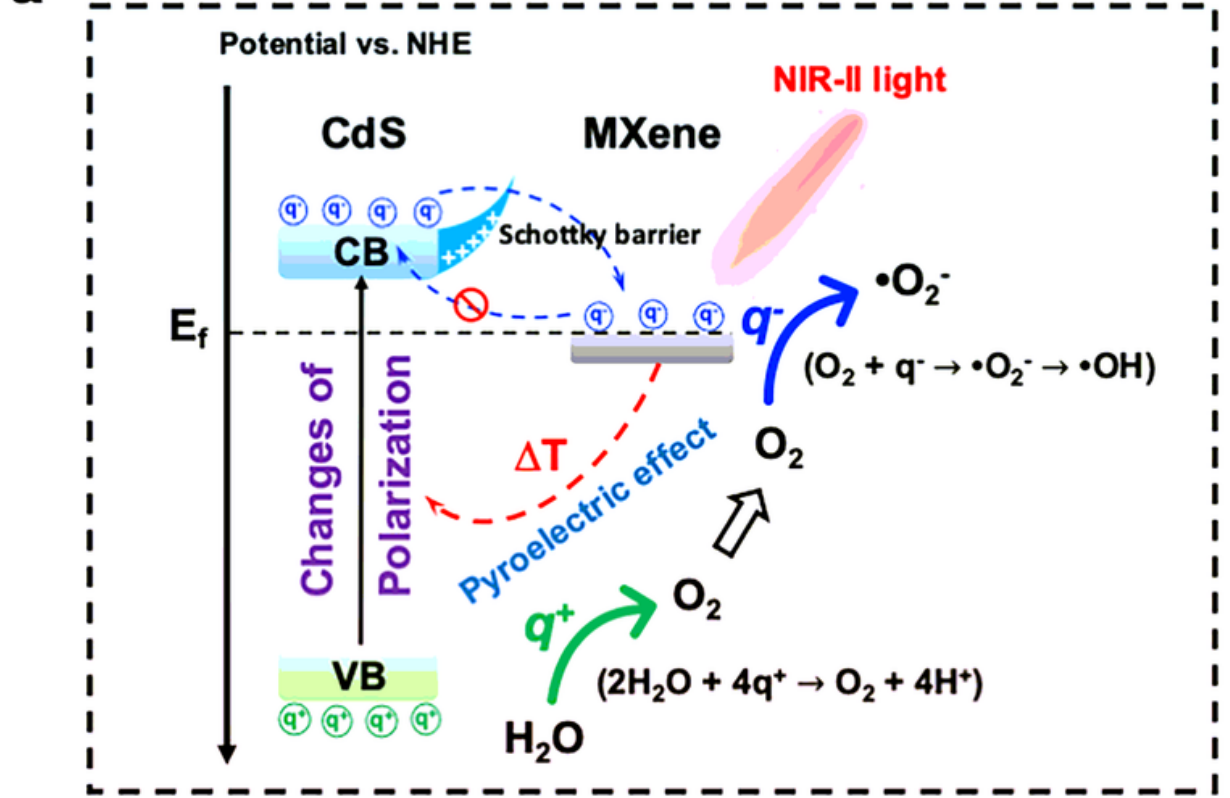

d

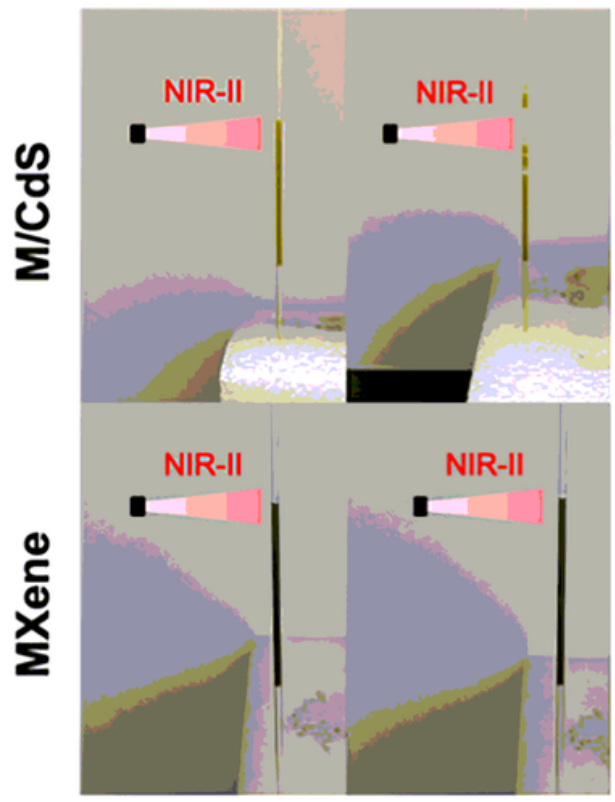

b

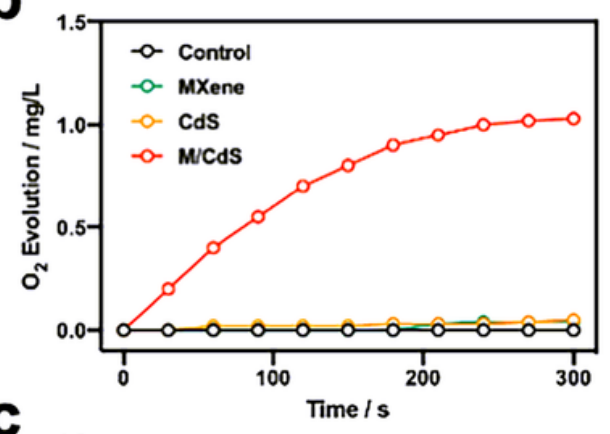

C

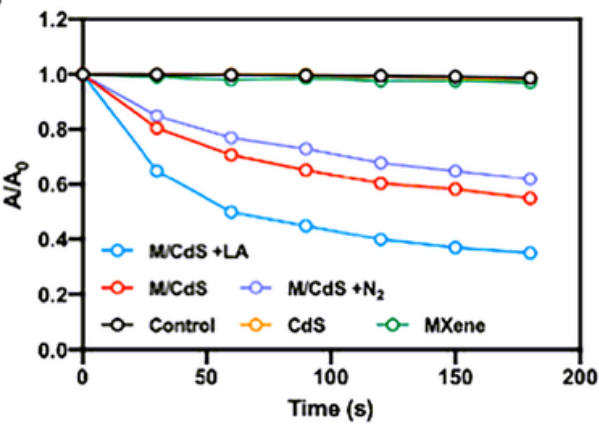

+ Laser

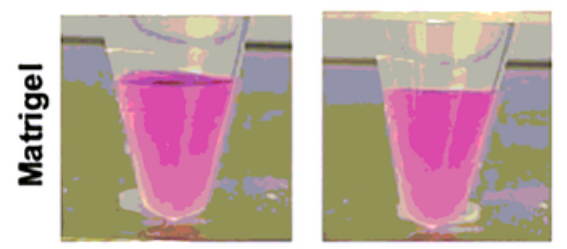

Origin

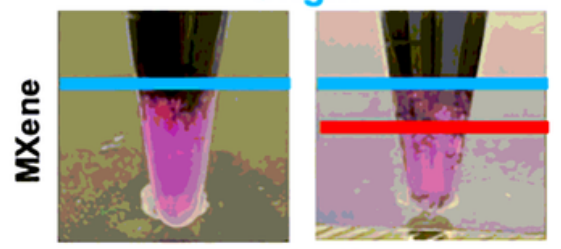

Penetration
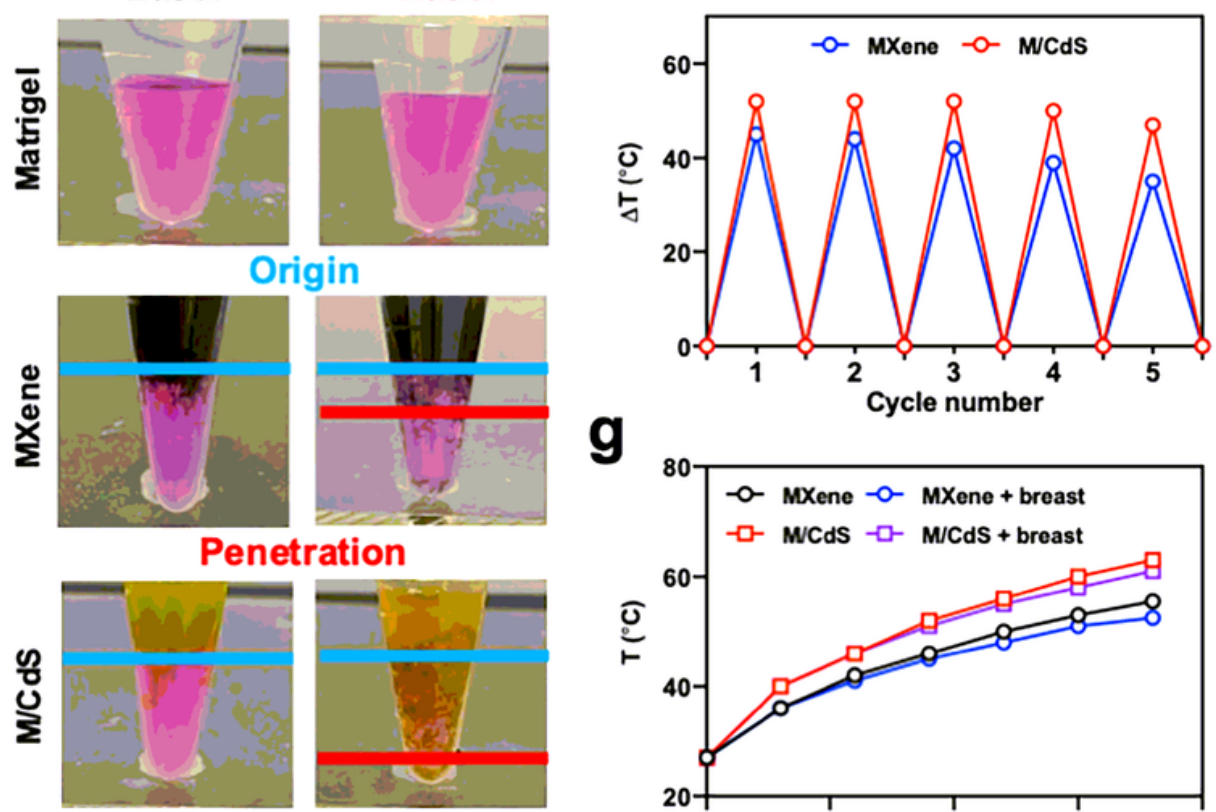

g

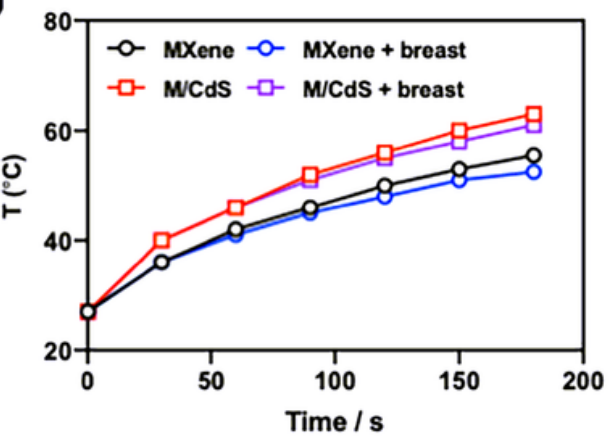

Figure 3

(a) Schematic illustration of pyroelectric effect and relationship between temperature variation (dT/dt) and pyroelectric current (I). EIS Nyquist plots of (b) CdS, (c) MXene and (d) M/CdS in dark and 1064-nm laser irradiation (1.0 W/cm2). (e) EIS Nyquist plots of $\mathrm{CdS}, \mathrm{MXene}$, and M/CdS under laser irradiation. (f) COMSOL finite element simulation of pyro-potential $\left(\Delta T=50^{\circ} \mathrm{C}\right)$ for CdS. (g) Photothermal effect of CdS, MXene, and M/CdS. (h) ITI of M/CdS under laser irradiation (3 min). (i) Pyroelectric currents of CdS and $\mathrm{M} / \mathrm{CdS}$ under laser irradiation. (j) Pyroelectric potentials of CdS and M/CdS under laser irradiation. MottSchottky curves of $(k)$ MXene and (l) CdS. 


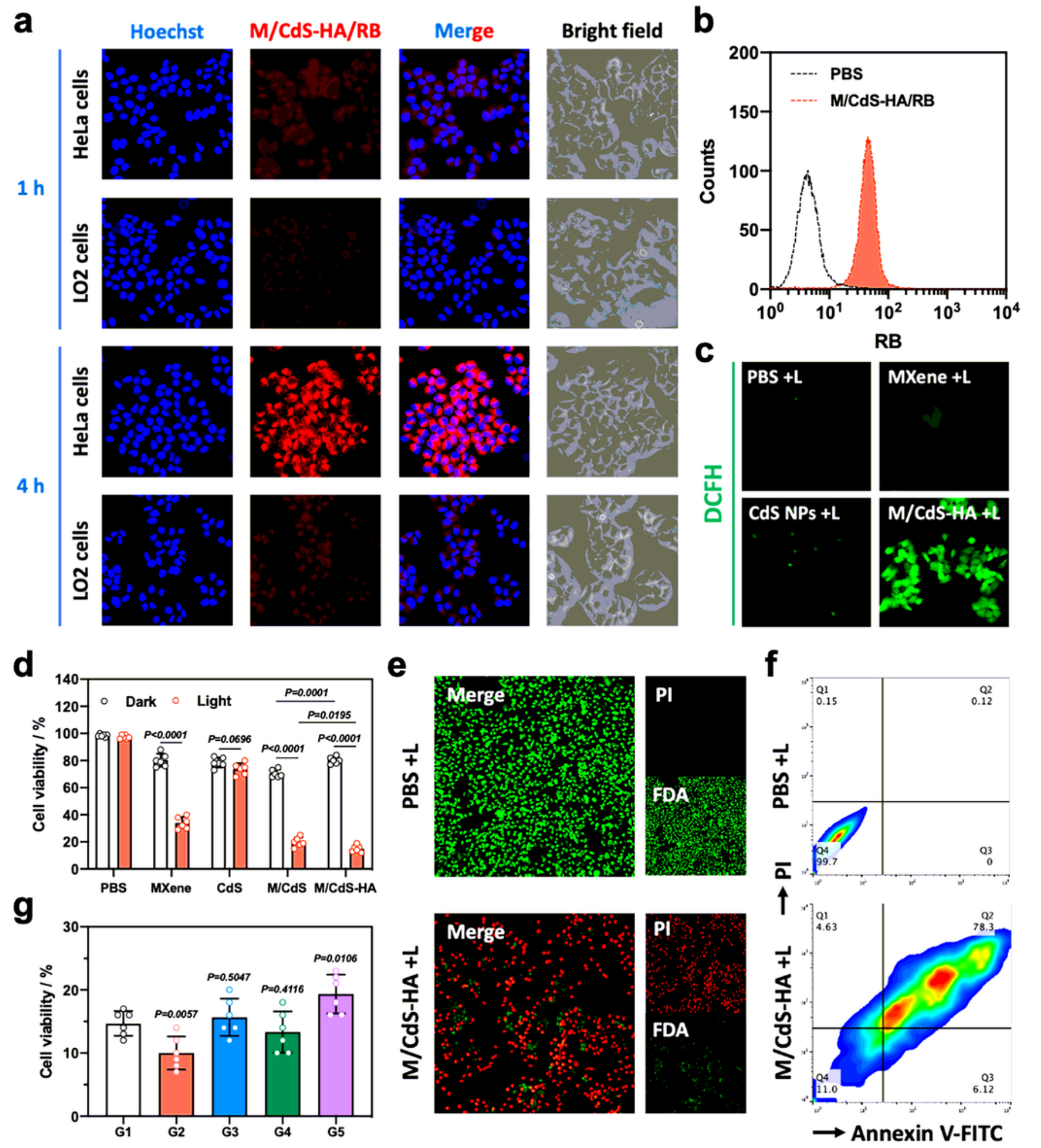

Figure 4

(a) Fluorescence images of HeLa and LO2 cells treated with M/CdS-HA/RB for one hour and four hours.

(b) FCM analysis of HeLa cells treated with PBS and M/CdS-HA/RB. (c) In vitro ROS generation of PBS $+\mathrm{L}$, MXene $+\mathrm{L}$, CdS NPs $+\mathrm{L}$, and M/CdS-HA $+\mathrm{L}$ tested by DCFH-DA. (d) Cell viability of HeLa cells treated with PBS, MXene, CdS, M/CdS, and M/CdS-HA in the dark and 1064-nm laser irradiation. (e) FDA/PI staining HeLa cells treated with PBS $+\mathrm{L}$ and M/CdS-HA $+\mathrm{L}$. (f) Cell apoptosis of HeLa cells treated with 
$P B S+L$ and $M / C d S-H A+L$. (g) Cell viability of HeLa cells treated with M/CdS-HA +L with G1: control, G2: LA (10 mM), G3: 5 mm chicken breast, G4: $\mathrm{NH4Cl}$ solution, and G5: hypoxia (P was calculated compared with $\mathrm{G} 1$ ).

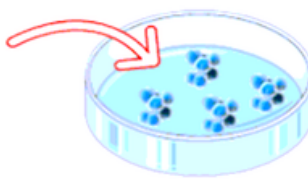

a

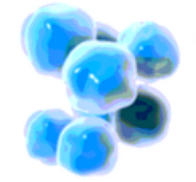

HeLa cells
Ultra-low attachment surface
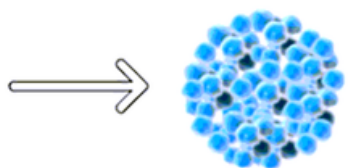

MCSs b
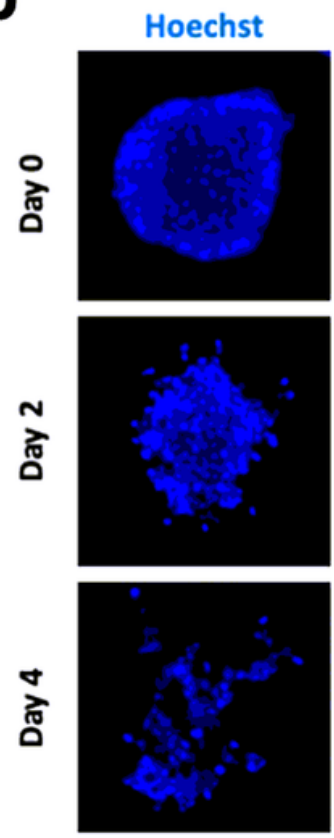

+Gen

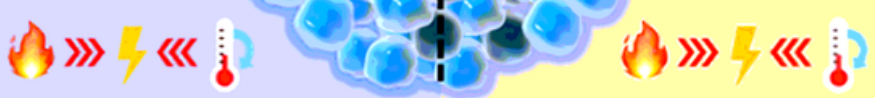

$\checkmark$ Diffusion (T个 + TIP $\downarrow)$

$\checkmark$ Transcytosis

$\checkmark$ Diffusion (T个 + TIP $\downarrow)$

X Transcytosis
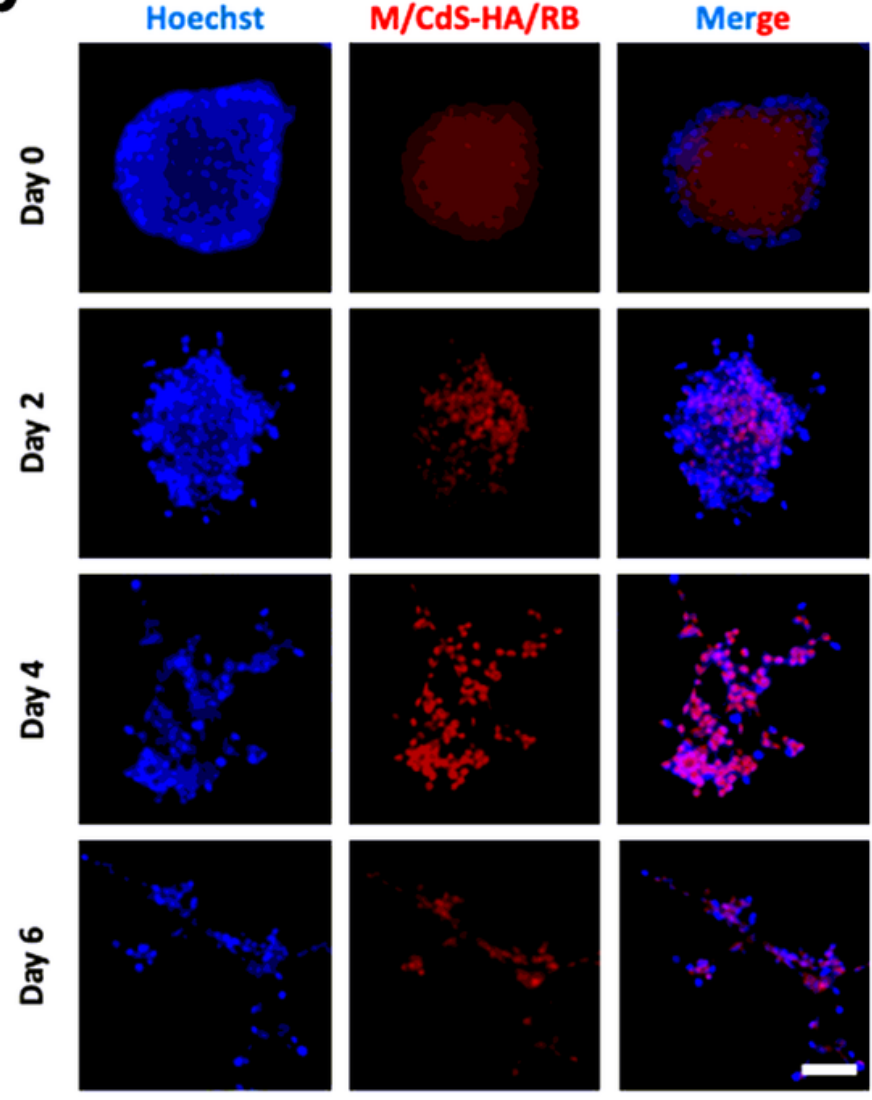

d $\mathrm{M} / \mathrm{CdS}+\mathrm{L}+\mathrm{Gen}$
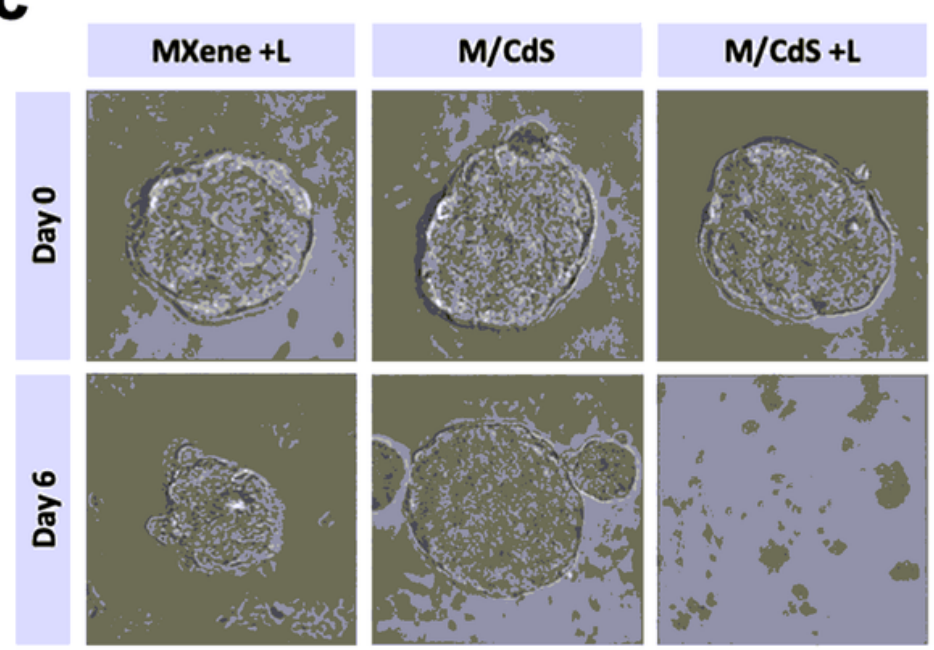
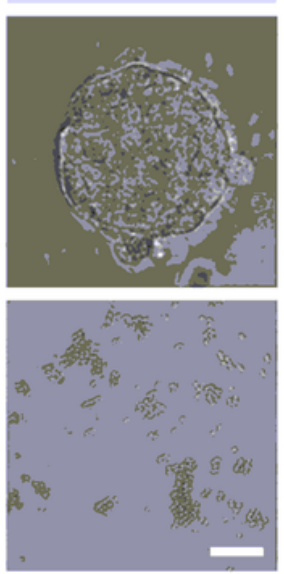

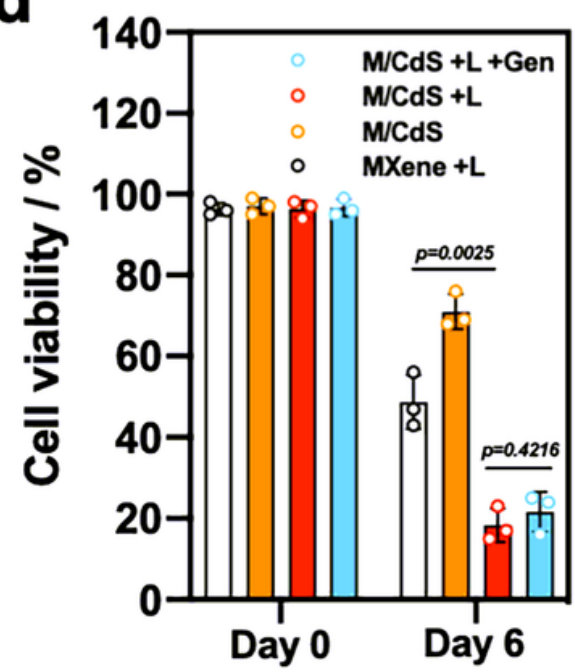

Figure 5

(a) Schematic illustration of MCSs formulation and penetration mechanism with enhanced diffusion by temperature rise $(T \uparrow)$, decrease of TIP, and transcytosis of MXene $+L, M / C d S, M / C d S+L$, and $M / C d S+L$ +Gen. (b) Fluorescence images of MCSs treated with M/CdS-HA/RB with 1064-nm laser irradiation on 
Day 0 to Day 6 (scale bar: $100 \mu \mathrm{m}$ ). (c) Images of MCSs treated with MXene $+\mathrm{L}, \mathrm{M} / \mathrm{CdS}, \mathrm{M} / \mathrm{CdS}+\mathrm{L}$, and $M / C d S+L+G e n$ on Day 0 and Day 6 (scale bar: $100 \mu \mathrm{m}$ ). (d) Cell viability of MCSs treated with MXene $+\mathrm{L}, \mathrm{M} / \mathrm{CdS}, \mathrm{M} / \mathrm{CdS}+\mathrm{L}$, and $\mathrm{M} / \mathrm{CdS}+\mathrm{L}+\mathrm{Gen}$ on Day 0 and Day 6 .

a

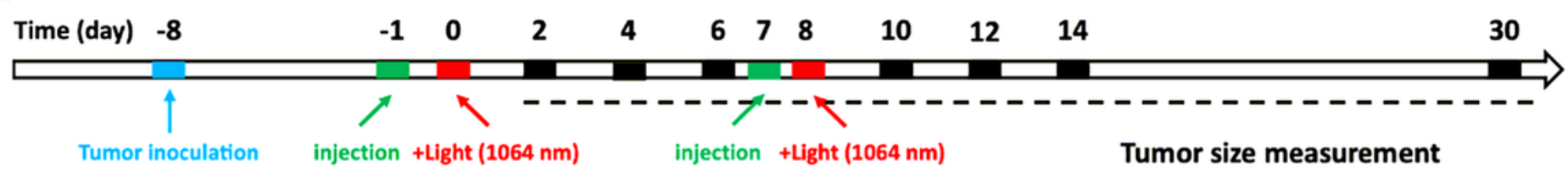

b

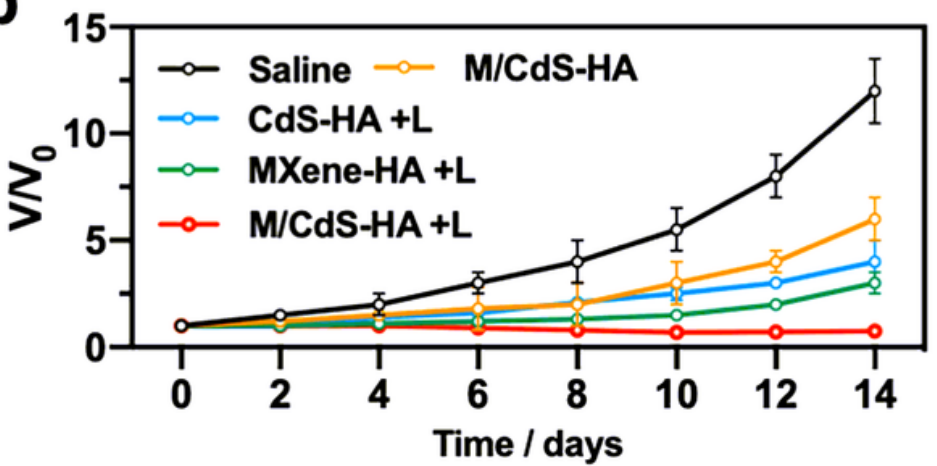

d

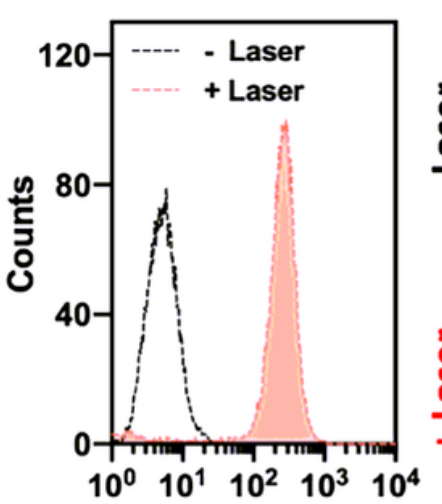

e

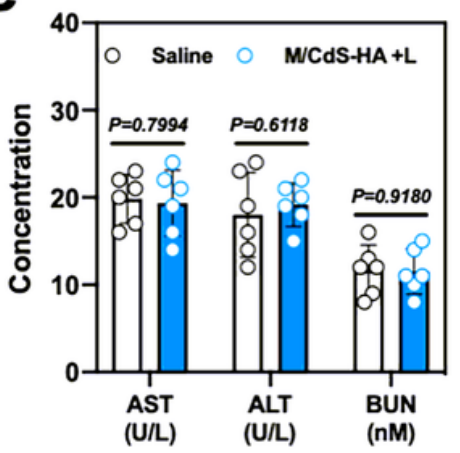

C
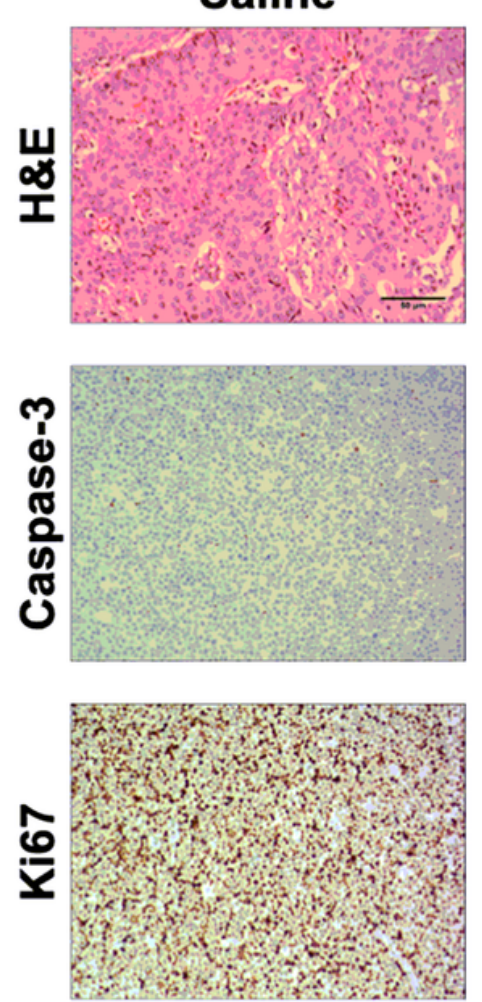

M/CdS-HA +L
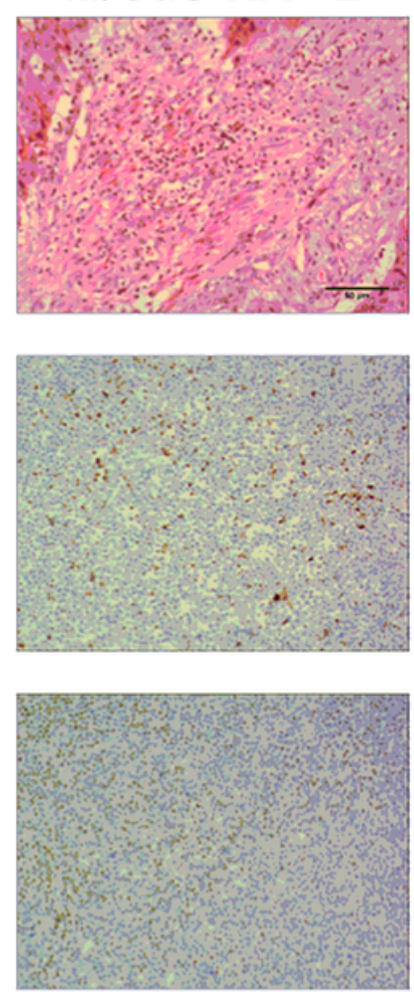

g

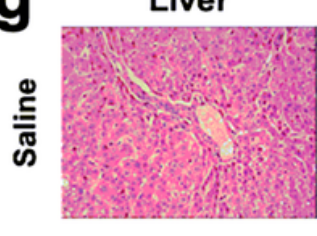

Spleen

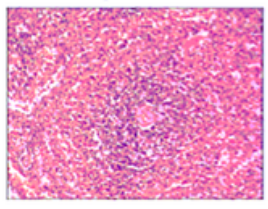

Kidney

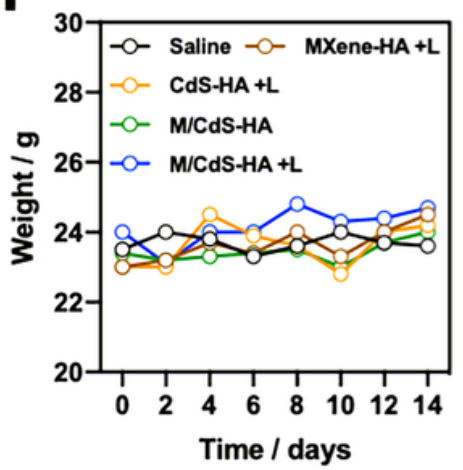

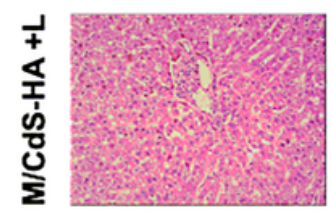

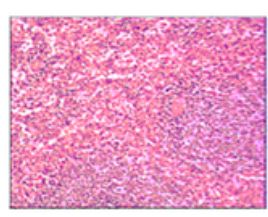

Figure 6

(a) Experimental illustration of antitumor therapy in vivo. (b) Tumor growth curves of all tested groups. (c) H\&E staining and immunohistochemical staining (Caspase-3 and Ki67) of Saline and M/CdS-HA +L. (d) ROS generation and photothermal effect in vivo of M/CdS-HA + L tested by FCM, DCF-FI, and ITI. (e) 
Serum biochemistry data of AST, ALT, and BUN. (f) Bodyweight changes of all tested groups. (g) Histological H\&E staining for major organs (liver, spleen, and kidney) from the mice treated with Saline and $\mathrm{M} / \mathrm{CdS}-\mathrm{HA}+\mathrm{L}$.

a

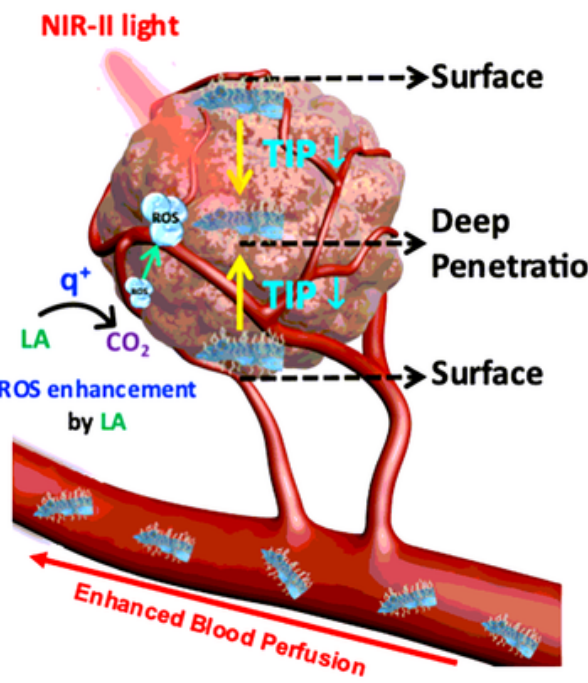

e

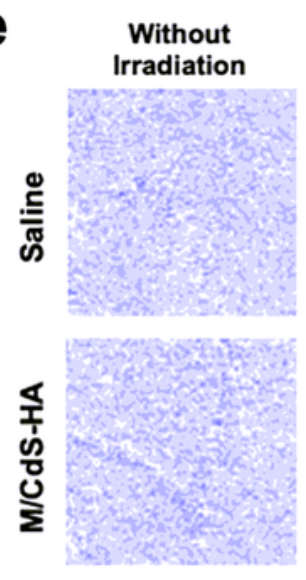

g
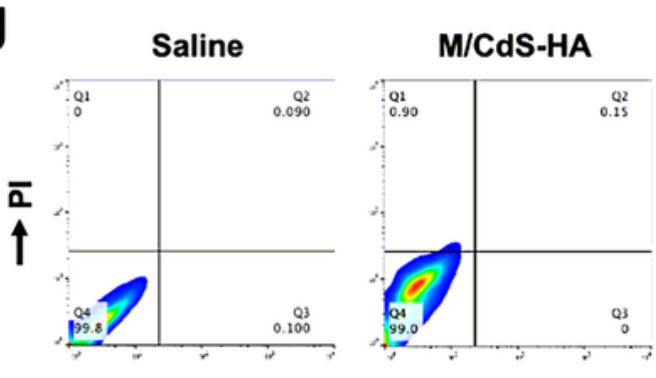

MXene-HA +L

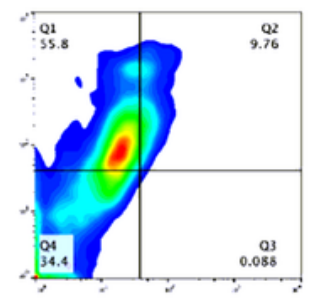

\section{Annexin V-FITC}

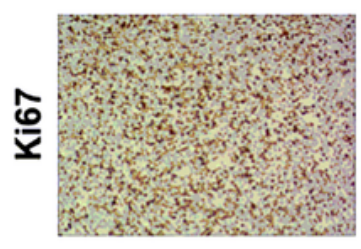

$15 \mathrm{~min}$ b
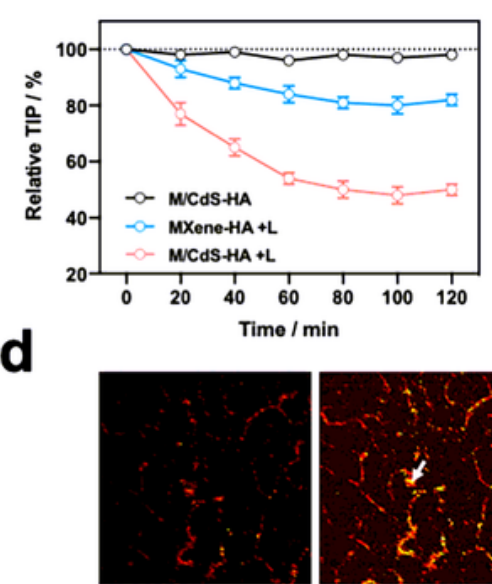

Without
Irradiation

ation

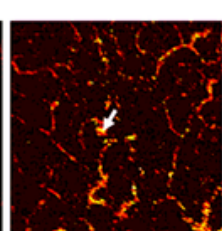

0 min

f
$30 \mathrm{~min}$
C

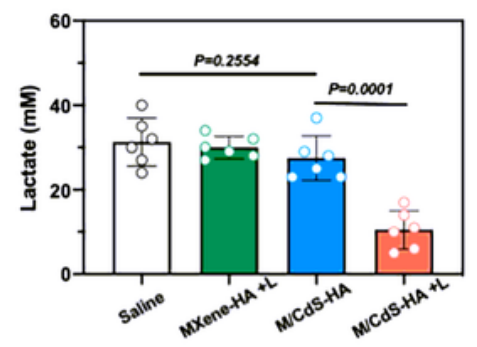

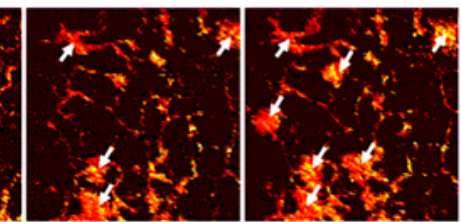

$15 \mathrm{~min}$

$30 \mathrm{~min}$

-nm Laser Irradiation
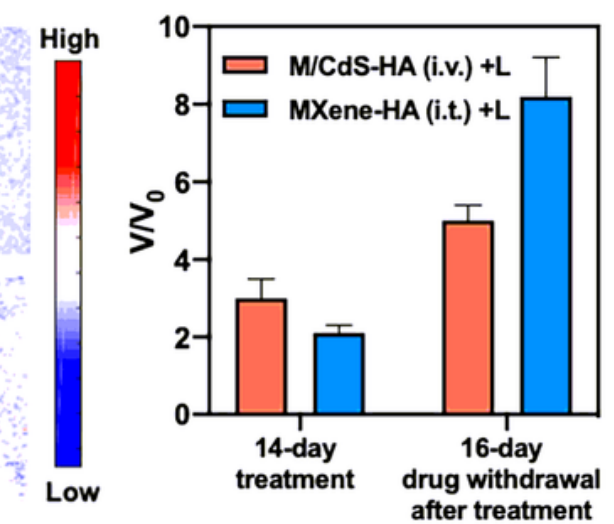

\section{Figure 7}

(a) Schematic illustration of the enhanced tumor penetration, blood perfusion by the decrease of TIP, and ROS enhancement by LA in the tumor. (b) The relative TIP values in the tumor treated with Saline, MXene- 
$\mathrm{HA}+\mathrm{L}, \mathrm{M} / \mathrm{CdS}-\mathrm{HA}$, and M/CdS-HA +L. (c) The contents of LA in the tumors treated with Saline, MXene-HA $+\mathrm{L}, \mathrm{M} / \mathrm{CdS}-\mathrm{HA}$, and M/CdS-HA $+\mathrm{L}$. (d) Photoacoustic images of the tumor blood vessels treated with M/CdS-HA (without/with 2 min of 1064-nm laser irradiation) at different time points (0 min, 15 min, and $30 \mathrm{~min}$ ). (e) Blood oxygen concentration in tumor vessels treated with Saline and M/CdS-HA (without/with $2 \mathrm{~min}$ of laser irradiation) at different time points ( $0 \mathrm{~min}, 15 \mathrm{~min}$, and $30 \mathrm{~min}$ ). (f) Tumor growth of MXene-HA (i.t.) $+L$ and M/CdS-HA (i.v.) $+L$ in the 14-day treatment and 16-day drug withdrawal after treatment. (g) Cell apoptosis and immunohistochemical staining (Ki67) for the center of tumor treated with Saline, M/CdS-HA, MXene-HA $+\mathrm{L}, \mathrm{M} / \mathrm{CdS}-\mathrm{HA}+\mathrm{L}$, and MXene-HA (i.t.) $+\mathrm{L}$.

\section{Supplementary Files}

This is a list of supplementary files associated with this preprint. Click to download.

- SupplementaryInformation.docx

- Scheme1.pdf 